HD-THEP-98-33

\title{
Differential cross sections for high energy elastic hadron-hadron scattering in nonperturbative $\mathrm{QCD}$
}

\author{
E. R. Berger ], O. Nachtmann? \\ Institut für Theoretische Physik \\ Universität Heidelberg \\ Philosophenweg 16 \\ 69120 Heidelberg, Germany
}

\begin{abstract}
Total and differential cross sections for high energy and small momentum transfer elastic hadron-hadron scattering are studied in QCD using a functional integral approach. The hadronic amplitudes are governed by vacuum expectation values of lightlike Wegner-Wilson loops, for which a matrix cumulant expansion is derived. The cumulants are evaluated within the framework of the Minkowskian version of the model of the stochastic vacuum. Using the second cumulant, we calculate elastic differential cross sections for hadron-hadron scattering. The agreement with experimental data is good.
\end{abstract}

\footnotetext{
${ }^{1}$ Supported by German Bundesministerium für Bildung und Forschung (BMBF), Contract Nr. 05 7HD $91 \mathrm{P}(0)$ and by Deutsche Forschungsgemeinschaft under grant no. GKR 216/1-98

${ }^{2}$ Email: E.Berger@thphys.uni-heidelberg.de

${ }^{3}$ Email: O.Nachtman@thphys.uni-heidelberg.de
} 


\section{Introduction}

In this article we will discuss elastic scattering of hadrons at high centre of mass energy $\sqrt{s}(\sqrt{s} \gtrsim 20 \mathrm{GeV})$ and low momentum transfer squared $t$ (say $\left.|t| \lesssim \mathrm{O}\left(1 \mathrm{GeV}^{2}\right)\right)$. Because of the small momentum transfer, such reactions are governed by soft, nonperturbative interactions. Experimental data show a rise for the total cross sections of all hadronic reactions [1] with increasing centre of mass (c.m.) energy, starting at about $\sqrt{\mathrm{s}}=10 \mathrm{GeV}$. Donnachie and Landshoff (DL) showed [2] that this rise can be described phenomenologically in terms of Regge theory [3] by pomeron exchange. The DL pomeron couples like a $C=+1$ "photon" to single quarks in the hadrons. The transition from the quark to the hadron level leads then to the additive quark rule [1. Donnachie and Landshoff fitted the rise of all hadronic cross sections with one small power of s, indicating that there is a universal mechanism which governs this kind of reactions. There is also a lot of data available for elastic differential cross sections at different c.m. energies, mainly for pp and $\mathrm{p} \overline{\mathrm{p}}$ scattering [5, 6, 7], but also for $\pi \mathrm{p}$ and Kp scattering [8]. Surely the mechanism which governs the elastic amplitude in the forward direction should also control the elastic differential cross section $(\mathrm{d} \sigma / d t)$ for sufficiently small $|t|$. Indeed, pomeron exchange is also able to describe $d \sigma / d t$ [9]. There are many other proposals and methods to describe such hadronic reactions, from perturbative field theoretic calculations [10], topological expansions and strings [11], valons [12], the work of Cheng and $\mathrm{Wu}$ on high energy behaviour in field theories based on perturbation theory [13], to "geometrical" models, which invoke global phenomenological properties of hadrons like their "blackness" [14. For a review of "pomeron physics" we refer to [15].

A new effort towards a microscopic description of high energy soft hadronic reactions was made in [16]. In an abelian gluon model the pomeron properties were related to nonperturbative properties of the vacuum like the gluon condensate [17] and a "vacuum correlation length" $a$ [18]. In [19] these ideas were generalised for QCD. It was shown there that the amplitude for qq-scattering at high energies is governed by the correlation function of two lightlike Wegner-Wilson lines. Using this formalism a description of hadron-hadron scattering was developed in [20, 21] where the hadronic amplitudes are calculated from correlation functions of lightlike Wegner-Wilson loops. These correlation functions are evaluated in the model of the stochastic vacuum 22], applied in Minkowski space after an analytic continuation from Euklidean space. There are by now many other applications of this technique, for example in the description of exclusive vector meson production [23]. Related techniques have been used in [24] for dealing with hard diffractive processes in deep inelastic lepton-nucleon scattering observed at HERA [25].

The goal of our paper is, to use and further develop the description of high energy diffractive hadron scattering given in [19, 21, 26, 27]. In Sect. 2 we collect the formulae for the hadronic scattering amplitudes as derived there. We begin Sect. 3 with a summary of the model of the stochastic vacuum (MSV) in its Minkowski version. In the second part of Sect. 3 we calculate the correlation function of two Wegner-Wilson loops, the main ingredient of the meson-meson scattering amplitude, using a matrix cumulant expansion 
and the MSV. A question of interest is whether or not the constituents of the baryons prefer quark-diquark like configurations where two quarks are close to each other on a scale given by the proton radius. In [28, 29] strong arguments for the quark-diquark picture were given where baryons do act in a first approximation as colour dipoles in the same way as mesons. In Sect. 4 we present our results for the $p p$ and $p \bar{p}$ elastic differential cross section $d \sigma / d t$ treating the baryons as such colour dipoles. In Sect. 5 we discuss meson-meson and meson-baryon scattering considering baryons again as colour dipoles. Treating meson-baryon and baryon-baryon scattering for general three-quark baryon configurations along these lines is more complicated and we defer this to another publication. Our conclusions are drawn in Sect. 6.

\section{The hadronic amplitudes}

Consider elastic scattering of two hadrons $h_{1}, h_{2}$ in the c.m. system at high energies and small momentum transfer

$$
h_{1}\left(P_{1}\right)+h_{2}\left(P_{2}\right) \rightarrow h_{1}\left(P_{3}\right)+h_{2}\left(P_{4}\right) .
$$

Now look at this reaction with a microscope. We have to choose an appropriate resolution in order to extract the essential features of the reaction, but not resolve unimportant details. In [19] this resolution was estimated, based on the uncertainty relation, with the following conclusions. Over a time interval $t_{0} \approx 2 \mathrm{fm}$ around the "nominal" interaction time (i) the parton state of the hadrons does not change qualitatively, i.e. parton annihilation and production processes can be neglected, (ii) partons travel in essence on straight lightlike world lines and (iii) the partons undergo "soft" elastic scattering governed by non-perturbative gluon dynamics. Using this approach hadronic amplitudes for high energies were derived in [21, 26]. The result for meson-meson scattering where mesons are represented as $q \bar{q}$ wave packets is

$$
\begin{aligned}
S_{f i}= & \delta_{f i}+i(2 \pi)^{4} \delta\left(P_{3}+P_{4}-P_{1}-P_{2}\right) T_{f i}, \\
T_{f i}= & (-2 i s) \int d^{2} b_{T} \exp \left(i \boldsymbol{q}_{T} \boldsymbol{b}_{T}\right) \int d^{2} x_{T} d^{2} y_{T} w_{3,1}^{M}\left(\boldsymbol{x}_{T}\right) w_{4,2}^{M}\left(\boldsymbol{y}_{T}\right) \\
& \left\langle W_{+}^{M}\left(\frac{1}{2} \boldsymbol{b}_{T}, \boldsymbol{x}_{T}\right) W_{-}^{M}\left(-\frac{1}{2} \boldsymbol{b}_{T}, \boldsymbol{y}_{T}\right)-1\right\rangle_{G} .
\end{aligned}
$$

Here the assumption is made that the $q$ and $\bar{q}$ share the longitudinal momentum of the meson roughly in equal proportions. The interpretation of (2) and the symbols occurring there is as follows. The scattering amplitude is obtained by first considering the scattering of quarks and antiquarks on a fixed gluon potential and then summing over all gluon potentials by path integration, indicated with the brackets \langle\rangle$_{G}$. Travelling through a gluon potential the quarks and antiquarks pick up non-abelian phase factors. To ensure gauge invariance the phase factors for $q$ and $\bar{q}$ from the same meson are joined at large positive and negative times, yielding lightlike Wegner-Wilson loops $W_{ \pm}$which are defined as

$$
W_{ \pm}^{M} \equiv \frac{1}{3} \operatorname{tr} V\left(C_{ \pm}\right),
$$




$$
V\left(C_{ \pm}\right)=\mathrm{P} \exp \left[-i g \int_{C_{ \pm}} d x^{\mu} G_{\mu}^{a}(x) \frac{\lambda^{a}}{2}\right] .
$$

Here $V\left(C_{ \pm}\right)$are non-abelian phase factors (connectors) along cut loops $C_{ \pm}$as sketched in Fig. 1. The trace in (3) corresponds in the usual way to the closure of the loop. The

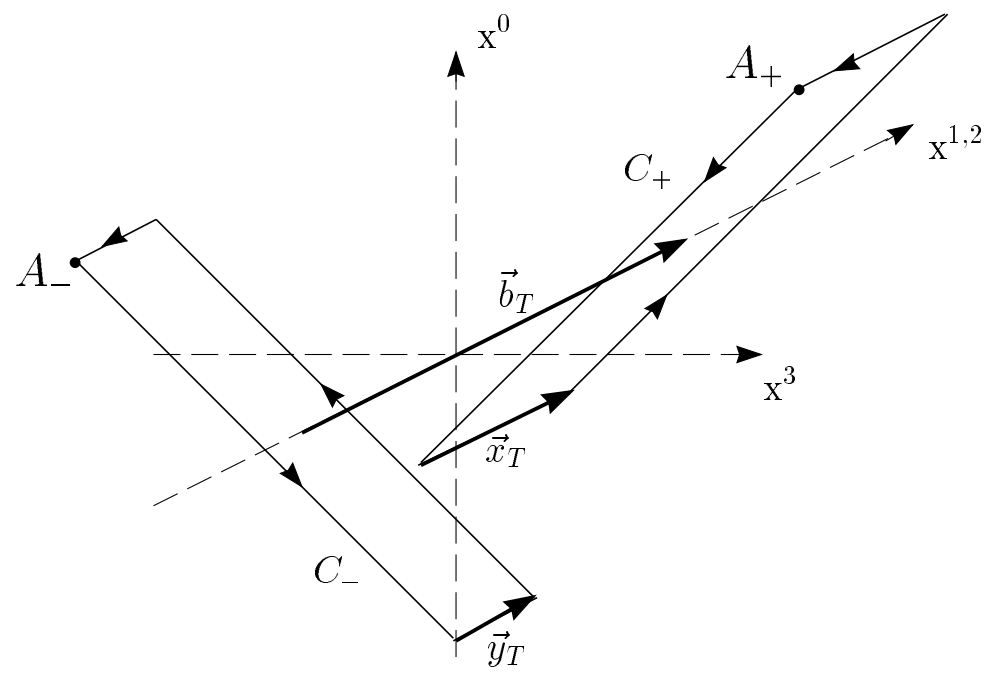

Figure 1: The light-like Wegner-Wilson cut loops in Minkowski space time, $C_{ \pm}$, consisting of two light like lines in the hyperplanes $x_{\mp}=0$ and connecting pieces at infinity. The loops are cut open at one corner, $A_{-}$and $A_{+}$, respectively. In transverse space the centres of the loops are at $\pm \boldsymbol{b}_{T} / 2$, the vectors from the antiquark to the quark lines are $\boldsymbol{x}_{T}$ and $\boldsymbol{y}_{T}$ respectively.

transverse separation between the centres of the loops is given by $\boldsymbol{b}_{T}$. The vectors $\boldsymbol{x}_{T}$ and $\boldsymbol{y}_{T}$ give the extensions and orientations of the loops in transverse space. The path integration correlates these loops and so causes the interaction. The resulting loop-loop correlation function has to be integrated over all extensions and orientations of the loops in transverse space with a measure given by the meson's overlap functions $w_{3,1}^{M}$ and $w_{4,2}^{M}$ for which one has to make a suitable ansatz. In order to obtain the hadronic amplitude a Fourier transform over the impact parameter $\boldsymbol{b}_{T}$ has to be done finally. In the following we call (2) the meson-meson amplitude. As discussed in the introduction, we can use it also to describe meson-baryon and baryon-baryon scattering treating baryons as colour dipoles in the quark-diquark picture.

\section{Evaluation of the meson-meson scattering ampli- tude}

In this section we will use the model of the stochastic vacuum (MSV) to perform the functional integral in (2) in an approximate way. 
First we give a short summary of the relevant properties of the MSV. For a detailed discussion see [21, 22]. The most important ingredient is a special ansatz for the correlation function of two parallel transported gluon field strength tensors, shifted to a common reference point $o$ along the curves $C_{x_{1}}$ and $C_{x_{2}}$.

$$
\left\langle\frac{g^{2}}{4 \pi^{2}} \hat{G}_{\mu \nu}^{a}\left(o, x_{1} ; C_{x_{1}}\right) \hat{G}_{\rho \sigma}^{b}\left(o, x_{2} ; C_{x_{2}}\right)\right\rangle \equiv \frac{1}{4} \delta^{a b} F_{\mu \nu \rho \sigma}\left(x_{1}, x_{2}, o ; C_{x_{1}}, C_{x_{2}}\right) .
$$

Here the right-hand side of (5) depends on $x_{1}, x_{2}$ and $C_{x_{1}}, C_{x_{2}}$. The reference point $o$ can be freely shifted on the curve $C_{12} \equiv C_{x_{1}}+\bar{C}_{x_{2}}$ where $\bar{C}_{x_{2}}$ is the oppositely oriented curve $C_{x_{2}}$. The correlation function is proportional to $\delta^{a b}$ due to colour conservation. Now the MSV makes the assumption, that $F_{\mu \nu \rho \sigma}$ is independent of the connecting curve $C_{12}$. Then Poincaré and parity invariance require the correlator to be of the following form $\left(z=x_{1}-x_{2}\right)$

$$
\begin{aligned}
F_{\mu \nu \rho \sigma}(z)= & \frac{1}{24} G_{2}\left\{\left(g_{\mu \rho} g_{\nu \sigma}-g_{\mu \sigma} g_{\nu \rho}\right)\left[\kappa D\left(z^{2}\right)+(1-\kappa) D_{1}\left(z^{2}\right)\right]\right. \\
& \left.+\left(z_{\sigma} z_{\nu} g_{\mu \rho}-z_{\rho} z_{\nu} g_{\mu \sigma}+z_{\rho} z_{\mu} g_{\nu \sigma}-z_{\sigma} z_{\mu} g_{\nu \rho}\right)(1-\kappa) \frac{\mathrm{d} D_{1}\left(z^{2}\right)}{\mathrm{d}\left(z^{2}\right)}\right\} .
\end{aligned}
$$

Here $G_{2}$ is the gluon condensate, $D$ and $D_{1}$ are invariant functions normalized to $D(0)=$ $D_{1}(0)=1$. For spacelike separations they are assumed to fall off rapidly on a length scale given by the correlation length $a \simeq 0.3 \mathrm{fm}$. The Fourier decomposition of the correlation functions is given by

$$
\begin{aligned}
D\left(z^{2}\right) & =\int_{-\infty}^{\infty} \frac{d^{4} k}{(2 \pi)^{4}} e^{-i k z} \tilde{D}\left(k^{2}\right) \\
D_{1}\left(z^{2}\right) & =\int_{-\infty}^{\infty} \frac{d^{4} k}{(2 \pi)^{4}} e^{-i k z} \tilde{D}_{1}\left(k^{2}\right) .
\end{aligned}
$$

We follow the authors of 21] and take as ansatz for $\tilde{D}$ and $\tilde{D}_{1}$

$$
\begin{aligned}
\tilde{D}\left(k^{2}\right) & =\frac{27(2 \pi)^{4}}{(8 a)^{2}} \frac{i k^{2}}{\left(k^{2}-\lambda^{-2}+i \epsilon\right)^{4}}, \\
\tilde{D}_{1}\left(k^{2}\right) & =\frac{2}{3} \frac{27(2 \pi)^{4}}{(8 a)^{2}} \frac{i}{\left(k^{2}-\lambda^{-2}+i \epsilon\right)^{3}} .
\end{aligned}
$$

The constant $\lambda$ is given by $\lambda=8 a / 3 \pi$ and $\kappa$ is a parameter related to the non-abelian character of the correlator [21, 31].

The Euclidean version of the correlator (5) has been investigated in lattice QCD [32]. The ansatz (7), (8) gives a good description of the nonperturbative part of the correlator in comparison to the data from the measurements in quenched QCD and from a fit one finds the following ranges for the parameters $G_{2}, a, \kappa$ [33]:

$$
\begin{aligned}
\kappa G_{2} a^{4} & =0.39 \text { to } 0.41 \\
\kappa & =0.80 \text { to } 0.89 \\
a & =0.33 \text { to } 0.37 \mathrm{fm} .
\end{aligned}
$$


From the lattice data one obtains directly the dimensionless quantities $\kappa$ and $\kappa G_{2} a^{4}$. Their uncertainty is due to statistical errors of the lattice data and to variations of the fit range chosen. The values for $a$ depend also on $\Lambda_{\text {Lattice }}$ which introduces some further uncertainty.

It was shown in [22] that $\kappa \neq 0$ is crucial for deriving confinement in the framework of the MSV. As was found for the total cross sections in [21] and as we will find again here a value $\kappa \neq 0$ is also crucial to describe the experimental data on high energy scattering for $d \sigma / d t$ in the framework of our model.

\subsection{A cumulant expansion of the colour dipole correlation func- tion}

Here we calculate the colour dipole correlation function $\left\langle W_{+}^{M} W_{-}^{M}\right\rangle_{G}$ introduced in (2). The strategy is the following. We transform the line integrals $W_{+}^{M}$ and $W_{-}^{M}$ into a surface

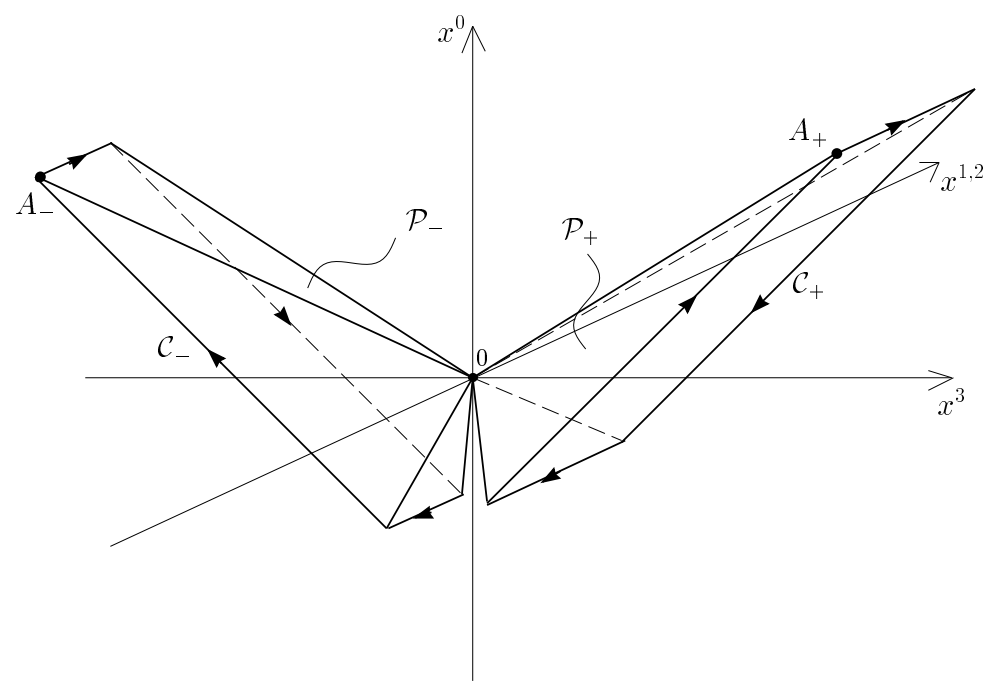

Figure 2: The curves $C_{+}$and $C_{-}$along which the connectors in $W_{ \pm}$are taken. The mantle of the pyramid with apex at the origin of the coordinate system and boundary $C_{+}\left(C_{-}\right)$is $P_{+}\left(P_{-}\right)$, the basis surface $S_{+}\left(S_{-}\right)$.

integral using the non-abelian Stokes theorem [34]. Following [21] we choose as surface the mantle of the double pyramid $P=P_{+}+P_{-}$which has $C_{+}+C_{-}$as boundary and $o$ as apex (Fig. 2).

To give the details, let $P_{ \pm}$be the pyramid surfaces excluding the base surfaces $S_{ \pm}$and cut from o to $A_{ \pm}$. Then the boundaries of $P_{ \pm}$are

$$
\begin{aligned}
& \partial P_{+}=o A_{+}+C_{+}+A_{+} o, \\
& \partial P_{-}=o A_{-}+C_{-}+A_{-} o .
\end{aligned}
$$

The methods developed for the non-abelian Stokes theorem as explained in [26] allow us 
to write the line integrals of (3) as

$$
\begin{aligned}
& V\left(C_{+}\right)=V\left(A_{+} O\right) V\left(P_{+}\right) V\left(o A_{+}\right), \\
& V\left(C_{-}\right)=V\left(A_{-} o\right) V\left(P_{-}\right) V\left(o A_{-}\right) .
\end{aligned}
$$

Here $V\left(o A_{ \pm}\right), V\left(A_{ \pm} O\right)$ are connectors along the straight lines from $A_{ \pm}$to $o$ and $o$ to $A_{ \pm}$, respectively. They satisfy

$$
\begin{aligned}
& V\left(o A_{+}\right) V\left(A_{+} O\right)=11 \\
& V\left(o A_{-}\right) V\left(A_{-} O\right)=11
\end{aligned}
$$

The matrices $V\left(P_{ \pm}\right)$in (11) are surface-ordered exponentials of field strength tensors $\hat{G}$ parallel transported to $O$ :

$$
\begin{aligned}
& V\left(P_{+}\right)=\mathrm{P} \exp \left[-\frac{i g}{2} \int_{P_{+}} d \sigma^{\mu \nu}(x) \hat{G}_{\mu \nu}^{a}\left(o, x ; C_{x}\right) \frac{\lambda^{a}}{2}\right] \\
& V\left(P_{-}\right)=\mathrm{P} \exp \left[-\frac{i g}{2} \int_{P_{-}} d \sigma^{\mu \nu}(x) \hat{G}_{\mu \nu}^{a}\left(o, x ; C_{x}\right) \frac{\lambda^{a}}{2}\right] .
\end{aligned}
$$

Inserting (11) in (2-3), using (12) and the cyclicity of the trace, we get

$$
\begin{aligned}
& \left\langle W_{+}^{M}\left(\frac{1}{2} \boldsymbol{b}_{T}, \boldsymbol{x}_{T}\right) W_{-}^{M}\left(-\frac{1}{2} \boldsymbol{b}_{T}, \boldsymbol{y}_{T}\right)\right\rangle_{G} \equiv\left\langle W_{+}^{M} W_{-}^{M}\right\rangle_{G} \\
& =\left\langle\frac{1}{3}\left[\operatorname{tr} V\left(C_{+}\right)\right] \frac{1}{3}\left[\operatorname{tr} V\left(C_{-}\right]\right\rangle_{G}\right. \\
& =\left\langle\frac{1}{3}\left[\operatorname{tr} V\left(P_{+}\right)\right] \frac{1}{3}\left[\operatorname{tr} V\left(P_{-}\right]\right\rangle_{G} .\right.
\end{aligned}
$$

The main idea is now to interpret the product of the two traces (tr) over $3 \times 3$ matrices in (14) as one trace $\left(\operatorname{Tr}_{2}\right)$ acting in the 9-dimensional tensor product space carrying the product of two $\mathrm{SU}(3)$ quark representations. Using the definition of the matrix multiplication in the product space giving e.g.

$$
\begin{aligned}
& \left(\lambda^{a} \otimes 1\right)\left(\lambda^{b} \otimes 1\right)=\lambda^{a} \lambda^{b} \otimes 1, \\
& \left(\lambda^{a} \otimes 1\right)\left(1 \otimes \lambda^{b}\right)=\lambda^{a} \otimes \lambda^{b}
\end{aligned}
$$

and of path ordering we get immediately

$$
\begin{aligned}
\left\langle W_{+}^{M} W_{-}^{M}\right\rangle_{G}=\frac{1}{9} \operatorname{Tr}_{2}\langle & \mathrm{P} \exp \left[-\frac{i g}{2} \int_{P_{+}} d \sigma^{\mu \nu} \hat{G}_{\mu \nu}^{a}\left(\frac{\lambda^{a}}{2} \otimes 1\right)\right] \\
& \left.\mathrm{P} \exp \left[-\frac{i g}{2} \int_{P_{-}} d \sigma^{\mu \nu} \hat{G}_{\mu \nu}^{a}\left(1 \otimes \frac{\lambda^{a}}{2}\right)\right]\right\rangle_{G} .
\end{aligned}
$$

The two exponentials in (16) commute because the two matrix structures in the exponents do. Introducing a total shifted field strength tensor $\hat{G}_{t}$ as

$$
\hat{G}_{t, \mu \nu}\left(o, x ; C_{x}\right)=\left\{\begin{array}{lll}
\hat{G}_{\mu \nu}^{a}\left(o, x ; C_{x}\right)\left(\frac{\lambda^{a}}{2} \otimes 1\right) & \text { for } & x \in P_{+} \\
\hat{G}_{\mu \nu}^{a}\left(o, x ; C_{x}\right)\left(1 \otimes \frac{\lambda^{a}}{2}\right) & \text { for } & x \in P_{-}
\end{array}\right.
$$


we can rewrite the two exponentials in (16) as one exponential defined in the direct product space. In this way we get from (14) a path-ordered integral over the double pyramid mantle $P=P_{+}+P_{-}$:

$$
\left\langle W_{+}^{M} W_{-}^{M}\right\rangle_{G}=\frac{1}{9} \operatorname{Tr}_{2}\left\langle\mathrm{P} \exp \left[-i \frac{g}{2} \int_{P} d \sigma(x) \hat{G}_{t}(x)\right]\right\rangle_{G} .
$$

Here and in the following we supress the Lorentz indices if there is no confusion. Note that the path orderings on $P_{+}$and $P_{-}$do not interfer with each other. Thus the path-ordering on $P$ can for instance be chosen such that all points of $P_{+}$are "later" than all points of $P_{-}$.

For the expectation value of the single surface ordered exponential (18) we can make a cumulant expansion [35, 26]. In our case we use a matrix cumulant expansion as explained in (2.41) of 26] (cf. also [36]):

$$
\left\langle\mathrm{P} \exp \left[-i \frac{g}{2} \int_{P} d \sigma(x) \hat{G}_{t}(x)\right]\right\rangle_{G}=\exp \left[\sum_{n=1}^{\infty} \frac{1}{n !}\left(-i \frac{g}{2}\right)^{n} \int d \sigma\left(x_{1}\right) \cdots d \sigma\left(x_{n}\right) K_{n}\left(x_{1}, . ., x_{n}\right)\right]
$$

Here the cumulants $K_{n}$ are functional integrals over products of the non-commuting matrices $\hat{G}_{t}$ of (17). Thus one has to be careful with their ordering. The cumulants up to $n=2$ are

$$
\begin{aligned}
K_{1}(x)= & \left\langle\hat{G}_{t}\left(o, x ; C_{x}\right)\right\rangle_{G}, \\
K_{2}\left(x_{1}, x_{2}\right)= & \left\langle\mathrm{P}\left[\hat{G}_{t}\left(o, x_{1} ; C_{x_{1}}\right) \hat{G}_{t}\left(o, x_{2} ; C_{x_{2}}\right)\right]\right\rangle_{G}- \\
& \frac{1}{2}\left(\left\langle\hat{G}_{t}\left(o, x_{1} ; C_{x_{1}}\right)\right\rangle_{G}\left\langle\hat{G}_{t}\left(o, x_{2} ; C_{x_{2}}\right)\right\rangle_{G}+(1 \leftrightarrow 2)\right) .
\end{aligned}
$$

Note that the $\hat{G}_{t}$ have Lorentz indices and are matrices in colour space as shown in (17). The functional integral indicated by \langle\rangle$_{G}$ in (20) involves only the field strength

components $\hat{G}_{\mu \nu}^{a}$, thus also the cumulants $K_{1}, K_{2}, \ldots$ still carry Lorentz and colour indices. The fact that there is no colour direction preferred in the vacuum requires $K_{1}$ to vanish. Neglecting cumulants higher than $n=2$ we get for (14)

$$
\begin{aligned}
& \left\langle W_{+}^{M} W_{-}^{M}\right\rangle_{G}=\frac{1}{9} \operatorname{Tr}_{2} \exp \left(C_{2}\left(\boldsymbol{x}_{T}, \boldsymbol{y}_{T}, \boldsymbol{b}_{T}\right)\right), \\
& C_{2}\left(\boldsymbol{x}_{T}, \boldsymbol{y}_{T}, \boldsymbol{b}_{T}\right)=-\frac{g^{2}}{8} \int_{P} d \sigma\left(x_{1}\right) \int_{P} d \sigma\left(x_{2}\right)\left\langle\mathrm{P}\left(\hat{G}_{t}\left(o, x_{1} ; C_{x_{1}}\right) \hat{G}_{t}\left(o, x_{2} ; C_{x_{2}}\right)\right)\right\rangle_{G} .
\end{aligned}
$$

where $C_{2}$ is a $9 \times 9$ matrix, invariant under $\mathrm{SU}(3)$ colour rotations.

\subsection{Calculation of the second cumulant term using the MSV}

Now we use the MSV with the ansatz (17),(8) for the invariant functions $D$ and $D_{1}$ to calculate $C_{2}$ of (21), which can be split into three contributions:

$$
C_{2}=C_{2}^{P_{+} P_{+}}+C_{2}^{P_{-} P_{-}}+C_{2}^{P_{+} P_{-}}
$$


In each of $C_{2}^{P_{+} P_{+}}$and $C_{2}^{P_{-} P_{-}}$both $x_{1}$ and $x_{2}$ move on the same surface, $P_{+}, P_{-}$, respectively, and so we have to pay attention to the surface ordering. In $C_{2}^{P_{+} P_{-}}$one point moves on $P_{+}$, the other one on $P_{-}$. In this case it follows from (17) that the shifted field strengths commute and the surface ordering is irrelevant for $C_{2}^{P_{+} P_{-}}$.

Now we show that $C_{2}^{P_{+} P_{+}}$vanishes. To see this we follow the argumentation of 26 and transform the two surface integrals over the pyramid mantle $P_{+}$in (21) into surface integrals over $S_{+}$and integrals over the volume $V_{+}$enclosed by $P_{+}$and $S_{+}$. The integrals over $S_{+}$vanish due to the Lorentz structure of the surface elements $d \sigma^{\mu \nu}$ together with the ansatz (6). The integrals over $V_{+}$are roughly speaking sums of integrals over surfaces parallel to $S_{+}$and so they vanish for the same reason. In a similar way we see that $C_{2}^{P_{-} P_{-}}$ vanishes.

To calculate $C_{2}^{P_{+} P_{-}}$we use the same method. In this case neither the integrals over $S_{ \pm}$ nor the ones over $V_{ \pm}$vanish. The integrations in light-like directions can be done analytically. Using then also the ordinary Gauss theorem we find with (5)-(7) that everything reduces to line integrals over the vectors $\boldsymbol{r}_{x i}, \boldsymbol{r}_{y i}(i=q, \bar{q})$ running from the apex $o$ to the position of the quarks and antiquarks in transverse space (Fig. 3):

$$
\begin{aligned}
& C_{2}\left(\boldsymbol{x}_{T}, \boldsymbol{y}_{T}, \boldsymbol{b}_{T}\right)=\frac{\lambda^{a}}{2} \otimes \frac{\lambda^{a}}{2}(-i) \chi\left(\boldsymbol{x}_{T}, \boldsymbol{y}_{T}, \boldsymbol{b}_{T}\right), \\
& \chi\left(\boldsymbol{x}_{T}, \boldsymbol{y}_{T}, \boldsymbol{b}_{T}\right)=\frac{G_{2} \pi^{2}}{24}\left\{I\left(\boldsymbol{r}_{x q}, \boldsymbol{r}_{y q}\right)+I\left(\boldsymbol{r}_{x \bar{q}}, \boldsymbol{r}_{y \bar{q}}\right)-\right. \\
& \left.I\left(\boldsymbol{r}_{x q}, \boldsymbol{r}_{y \bar{q}}\right)-I\left(\boldsymbol{r}_{x \bar{q}}, \boldsymbol{r}_{y q}\right)\right\} \\
& I\left(\boldsymbol{r}_{x}, \boldsymbol{r}_{y}\right)=i \int_{0}^{1} d v_{1} \int_{0}^{1} d v_{2} \int_{-\infty}^{\infty} \frac{d^{2} k_{T}}{(2 \pi)^{2}} e^{i \boldsymbol{k}_{T}\left(v_{1} \boldsymbol{r}_{y}-v_{2} \boldsymbol{r}_{x}\right)} \\
& \left\{\kappa \boldsymbol{r}_{y} \boldsymbol{r}_{x} \tilde{D}\left(-\boldsymbol{k}_{T}^{2}\right)+(1-\kappa)\left(\boldsymbol{k}_{T} \boldsymbol{r}_{y}\right)\left(\boldsymbol{k}_{T} \boldsymbol{r}_{x}\right) \tilde{D}_{1}^{\prime}\left(-\boldsymbol{k}_{T}^{2}\right)\right\}, \\
& \tilde{D}_{1}^{\prime}\left(k^{2}\right)=\frac{d}{d k^{2}} \tilde{D}_{1}\left(k^{2}\right) \text {. }
\end{aligned}
$$

As we can see we finally need the correlator functions $\tilde{D}, \tilde{D}_{1}$ of (间) for space-like momenta only. This means that the result (23) involves the correlation functions $D\left(z^{2}\right)$ and $D_{1}\left(z^{2}\right)$ only for spacelike $z$, where they are as in Euclidean space time. With (8) for the functions $\tilde{D}, \tilde{D}_{1}$ we get

$$
\begin{aligned}
I\left(\boldsymbol{r}_{x}, \boldsymbol{r}_{y}\right)= & \left\{\kappa \frac { \pi } { 2 } \lambda ^ { 2 } ( \boldsymbol { r } _ { y } \boldsymbol { r } _ { x } ) \int _ { 0 } ^ { 1 } d v \left(\left(\frac{\left|v \boldsymbol{r}_{y}-\boldsymbol{r}_{x}\right|}{\lambda}\right)^{2} K_{2}\left(\frac{\left|v \boldsymbol{r}_{y}-\boldsymbol{r}_{x}\right|}{\lambda}\right)+\right.\right. \\
& \left.\left(\frac{\left|\boldsymbol{r}_{y}-v \boldsymbol{r}_{x}\right|}{\lambda}\right)^{2} K_{2}\left(\frac{\left|\boldsymbol{r}-v \boldsymbol{r}_{x}\right|}{\lambda}\right)\right) \\
& +(1-\kappa) \pi \lambda^{4}\left(\left(\frac{\left|\boldsymbol{r}_{y}-\boldsymbol{r}_{x}\right|}{\lambda}\right)^{3} K_{3}\left(\frac{\left|\boldsymbol{r}_{y}-\boldsymbol{r}_{x}\right|}{\lambda}\right)\right\}
\end{aligned}
$$

where $K_{2,3}$ are the modified Bessel functions. Note that $\chi$ is a real function. Inserting (23) in (21) we obtain 


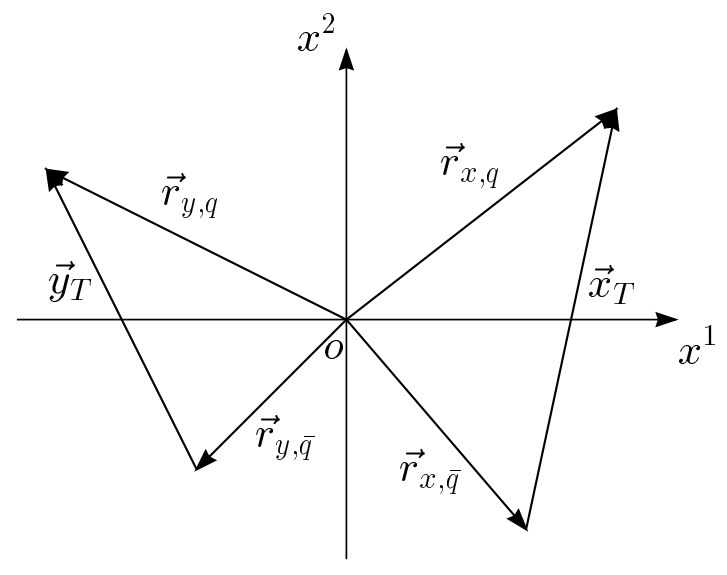

Figure 3: The projection of the pyramid surfaces $P_{ \pm}+S_{ \pm}$into transverse space. The vectors $\boldsymbol{r}_{x, q(\bar{q})}$ and $\boldsymbol{r}_{y, q(\bar{q})}$ point from the origin o along the transverse projections of the pyramid mantles $P_{ \pm}$to the projections of the quark and antiquark lines of $C_{ \pm} \cdot \boldsymbol{x}_{T}$ and $\boldsymbol{y}_{T}$ point from the antiquark to the quark lines.

$$
\begin{aligned}
& \left\langle W_{+}^{M}\left(\frac{1}{2} \boldsymbol{b}_{T}, \boldsymbol{x}_{T}\right) W_{-}^{M}\left(-\frac{1}{2} \boldsymbol{b}_{T}, \boldsymbol{y}_{T}\right)\right\rangle_{G}= \\
& \frac{1}{9} \operatorname{Tr}_{2} \exp \left[\left(\frac{\lambda^{a}}{2} \otimes \frac{\lambda^{a}}{2}\right)(-i) \chi\left(\boldsymbol{x}_{T}, \boldsymbol{y}_{T}, \boldsymbol{b}_{T}\right)\right] .
\end{aligned}
$$

To evaluate the trace in (25) we introduce two projectors $P_{s}$ and $P_{a}$

$$
\begin{aligned}
& \left(P_{s}\right)_{\left(\alpha_{1} \alpha_{2}\right)\left(\beta_{1} \beta_{2}\right)}=\frac{1}{2}\left(\delta_{\alpha_{1} \beta_{1}} \delta_{\alpha_{2} \beta_{2}}+\delta_{\alpha_{1} \beta_{2}} \delta_{\alpha_{2} \beta_{1}}\right), \\
& \left(P_{a}\right)_{\left(\alpha_{1} \alpha_{2}\right)\left(\beta_{1} \beta_{2}\right)}=\frac{1}{2}\left(\delta_{\alpha_{1} \beta_{1}} \delta_{\alpha_{2} \beta_{2}}-\delta_{\alpha_{1} \beta_{2}} \delta_{\alpha_{2} \beta_{1}}\right)
\end{aligned}
$$

which act in the direct product space of two $\mathrm{SU}(3)$ quark representations projecting onto the subspaces carrying the irreducible representations. The decomposition is: $3 \otimes 3=6 \oplus \overline{3}$. Using the identity:

$$
\frac{\lambda^{a}}{2} \otimes \frac{\lambda^{a}}{2}=\frac{1}{3} P_{s}-\frac{2}{3} P_{a},
$$

together with the projector properties of $P_{s}$ and $P_{a}$ and $\operatorname{Tr}_{2} P_{s}=6$ and $\operatorname{Tr}_{2} P_{a}=3$ we can immediately calculate the trace in (25) and so the colour dipole correlation function:

$$
\begin{aligned}
& \operatorname{Tr}_{2} \exp \left[\left(\frac{\lambda^{a}}{2} \otimes \frac{\lambda^{a}}{2}\right)(-i \chi)\right] \\
& =\operatorname{Tr}_{2} \exp \left[\left(\frac{1}{3} P_{s}-\frac{2}{3} P_{a}\right)(-i \chi)\right] \\
& =\operatorname{Tr}_{2}\left[P_{s} e^{-i \frac{1}{3} \chi}+P_{a} e^{i \frac{2}{3} \chi}\right] \\
& =6 e^{-i \frac{1}{3} \chi}+3 e^{i \frac{2}{3} \chi}
\end{aligned}
$$




\subsection{The meson-meson amplitude}

Inserting (28) in (2) and using the Gaussian shaped mesonic overlap functions from [21] our final result for the meson-meson scattering amplitude reads

$$
\begin{aligned}
& T_{f i}=(2 i s)(2 \pi) \int_{0}^{\infty} d b b J_{0}(\sqrt{|t|} b) \hat{J}_{M, M}(b), \\
& \hat{J}_{M, M}(b)=-\int d^{2} x_{T} \frac{1}{2 \pi S_{H_{1}}^{2}} \exp \left(-\frac{\boldsymbol{x}_{T}^{2}}{2 S_{H_{1}}^{2}}\right) \int d^{2} y_{T} \frac{1}{2 \pi S_{H_{2}}^{2}} \exp \left(-\frac{\boldsymbol{y}_{T}^{2}}{2 S_{H_{2}}^{2}}\right) \\
&\left\{\frac{2}{3} \cos \left(\frac{1}{3} \chi\left(\boldsymbol{x}_{T}, \boldsymbol{y}_{T}, \boldsymbol{b}_{T}\right)\right)+\frac{1}{3} \cos \left(\frac{2}{3} \chi\left(\boldsymbol{x}_{T}, \boldsymbol{y}_{T}, \boldsymbol{b}_{T}\right)\right)+\right. \\
& i {\left.\left[-\frac{2}{3} \sin \left(\frac{1}{3} \chi\left(\boldsymbol{x}_{T}, \boldsymbol{y}_{T}, \boldsymbol{b}_{T}\right)\right)+\frac{1}{3} \sin \left(\frac{2}{3} \chi\left(\boldsymbol{x}_{T}, \boldsymbol{y}_{T}, \boldsymbol{b}_{T}\right)\right)\right]-1\right\} . }
\end{aligned}
$$

Here $J_{0}$ is the zeroth-order Bessel function. Due to (28) we get from the original matrix valued exponential (21) a sum of two c-number valued exponentials which we have written in terms of trigonometric functions. Different hadrons are distinguished in (29) only through their strong interaction extension parameters $S_{H}$ which should be of the order of the electromagnetic hadron radii.

Assuming $|\chi| \ll 1$ and expanding $\hat{J}_{M, M}(b)$ in (29) to the order $O\left(\chi^{2}\right)$ gives the result of 21.

$$
\begin{aligned}
T_{f i}=(2 i s) & (2 \pi) \int_{0}^{\infty} d b b J_{0}(\sqrt{|t|} b) \hat{J}_{M, M}^{(2)}(b), \\
\hat{J}_{M, M}^{(2)}(b)= & \int d^{2} x_{T} \frac{1}{2 \pi S_{H_{1}}^{2}} \exp \left(-\frac{\boldsymbol{x}_{T}^{2}}{2 S_{H_{1}}^{2}}\right) \int d^{2} y_{T} \frac{1}{2 \pi S_{H_{2}}^{2}} \exp \left(-\frac{\boldsymbol{y}_{T}^{2}}{2 S_{H_{1}}^{2}}\right) \\
& \cdot \frac{1}{9}\left(\chi\left(\boldsymbol{x}_{T}, \boldsymbol{y}_{T}, \boldsymbol{b}_{T}\right)\right)^{2},
\end{aligned}
$$

But the integral $\hat{J}_{M, M}(b)$ in $(30)$ is dominated by a region in $\boldsymbol{x}_{T}, \boldsymbol{y}_{T}$ where $|\chi| \ll 1$ only for larger values of $b$, say $b>4 a$ (see section 4 ). So using (30) $\hat{J}_{M, M}(b)$ can be calculated reliably only for larger values of $b$. Nevertheless with (30) the total cross section and the slope parameter at $t=0$, where one needs only the first and third moments of $\hat{J}_{M, M}(b)$, were calculated in [21] in a satisfactory way.

Coming back to our expression (29) for the elastic scattering amplitude we show next that this amplitude is purely imaginary. Since $\chi$ is real, any real part of (29) would have to come from the sine-terms. Now the overlap functions in (29) are invariant under the replacements: $\boldsymbol{x}_{T} \rightarrow-\boldsymbol{x}_{T}$ and $\boldsymbol{y}_{T} \rightarrow-\boldsymbol{y}_{T}$, respectively, but

$$
\chi\left(-\boldsymbol{x}_{T}, \boldsymbol{y}_{T}, \boldsymbol{b}_{T}\right)=\chi\left(\boldsymbol{x}_{T},-\boldsymbol{y}_{T}, \boldsymbol{b}_{T}\right)=-\chi\left(\boldsymbol{x}_{T}, \boldsymbol{y}_{T}, \boldsymbol{b}_{T}\right) .
$$

This is easily seen from (23) since $\boldsymbol{x}_{T} \rightarrow-\boldsymbol{x}_{T}$ means the replacement $\boldsymbol{r}_{x q} \leftrightarrow r_{x \bar{q}}$ and $\boldsymbol{y}_{T} \rightarrow-\boldsymbol{y}_{T}$ the replacement $\boldsymbol{r}_{y q} \leftrightarrow \boldsymbol{r}_{y \bar{q}}$. Thus integrating over $\boldsymbol{x}_{T}$ and $\boldsymbol{y}_{T}$ averages out 
the real part in (29) and we get

$$
\begin{aligned}
& T_{f i}=(2 i s)(2 \pi) \int_{0}^{\infty} d b b J_{0}(\sqrt{|t|} b) \hat{J}_{M, M}(b), \\
& \hat{J}_{M, M}(b)=-\int d^{2} x_{T} \frac{1}{2 \pi S_{H_{1}}^{2}} \exp \left(-\frac{\boldsymbol{x}_{T}^{2}}{2 S_{H_{1}}^{2}}\right) \int d^{2} y_{T} \frac{1}{2 \pi S_{H_{2}}^{2}} \exp \left(-\frac{\boldsymbol{y}_{T}^{2}}{2 S_{H_{2}}^{2}}\right) \\
& {\left[\frac{2}{3} \cos \left(\frac{1}{3} \chi\left(\boldsymbol{x}_{T}, \boldsymbol{y}_{T}, \boldsymbol{b}_{T}\right)\right)+\frac{1}{3} \cos \left(\frac{2}{3} \chi\left(\boldsymbol{x}_{T}, \boldsymbol{y}_{T}, \boldsymbol{b}_{T}\right)\right)-1\right] . }
\end{aligned}
$$

As a consequence our meson-meson amplitude is invariant under the replacement of one hadron by its antihadron. The exchange of all partons by their antipartons for a given parton configuration turns around the loop direction. This results in a change of sign of $\chi$ and so does not affect the amplitude (32). In our approximations, we get only charge conjugation $C=+1$ (pomeron) exchange and no $C=-1$ (odderon) exchange contributions to the amplitude. A real part of the amplitude and $C=-1$ exchange contributions could arise from higher cumulants in (19).

We discuss now the constraints on the elastic amplitude implied by the partial wave unitarity (see for instance [39]). The partial wave expansion for the $T$ matrix element for spin-zero mesons reads

$$
\begin{gathered}
T(s, t)=\frac{8 \pi \sqrt{s}}{P_{c m}} \sum_{l=0}^{\infty}(2 l+1) P_{l}(\cos \vartheta) a_{l}(s), \\
a_{l}(s)=\frac{1}{2 i}\left(e^{2 i \delta_{l}} \eta_{l}-1\right) \\
0 \leq \eta_{l} \leq 1 .
\end{gathered}
$$

Here $P_{l}$ are the Legendre polynomials, $P_{c m}$ is the $\mathrm{cm}$ momentum, $\vartheta$ the $\mathrm{cm}$ scattering angle, and $\delta_{l}, \eta_{l}$ are the phase shifts and inelasticities, respectively.

At high energies we get from (33) with $b=(2 l+1) / \sqrt{s}$ :

$$
T(x, t)=8 \pi s \int_{0}^{\infty} d b b J_{0}(b \sqrt{|t|}) a_{l}(\sqrt{s}) .
$$

Comparison with (32) gives

$$
\hat{J}_{M M}(b)=\left.\left[-e^{2 i \delta_{l}} \eta_{l}+1\right]\right|_{2 l+1=b \sqrt{s}} .
$$

Thus, the partial wave unitarity requires

$$
\left|\hat{J}_{M M}(b)-1\right| \leq 1
$$

and this is always satisfied for our amplitude (32) since $\chi$ is real and the profile functions are normalised to one. 


\section{Proton-proton and proton-antiproton scattering in the quark-diquark picture}

A lot of experimental data is available for elastic differential cross sections of protonproton $(p p)$ scattering up to $\sqrt{s}=63 \mathrm{GeV}$ and proton-antiproton $(p \bar{p})$ scattering up to Tevatron energies of $\sqrt{s}=1800 \mathrm{GeV}$ [5, 6, 7]. In this section we will compare the high energy data in the range $\sqrt{s} \geq 23 \mathrm{GeV}$ to the results of our calculations making the assumption that the proton has a quark-diquark structure. Thus we can use the formulae for the meson-meson amplitude as presented in Sect. 3.

Our starting point is (32) depending on 4 parameters: the QCD vacuum parameters $G_{2}, \kappa$ and $a$ and the proton extension parameter $S_{H_{1}}=S_{H_{2}}=S_{p}$. The vacuum parameters are surely energy and process independent, the extension parameter $S_{p}$ will be allowed to vary with energy. The numerical calculations using (32) are too lengthy to attempt a "best fit" of these parameters from the data. We adopted the following procedure instead. In the SVM with the ansatz (7,8) for the functions $D, D_{1}$ the string tension $\rho$ is given by

$$
\begin{aligned}
\rho & =\frac{\pi^{3} \kappa G_{2}}{36} \int_{0}^{\infty} d Z^{2} D\left(-Z^{2}\right) \\
& =\frac{32 \pi \kappa G_{2} a^{2}}{81} .
\end{aligned}
$$

Typical values for $\rho$ extracted from phenomenology (cf. e.g. [37]) are $\rho=(420 \pm 20 \mathrm{MeV})^{2}$. In the following we will express $G_{2}$ through $\rho$ and $a$ using (38). From previous work 21, 30 and the lattice measurements discussed in Sect. 3 we expect for the correlation length $0.30 \mathrm{fm} \lesssim a \lesssim 0.37 \mathrm{fm}$, for $\kappa \approx 0.75$ and for the proton extension parameter at $\sqrt{s}=23$ $\mathrm{GeV} S_{p} \approx 0.86$ corresponding to the electromagnetic proton radius. Now we considered again $\sqrt{s}=23 \mathrm{GeV}$ and started our numerical investigations using (32), varying the parameters $\rho, a, \kappa, S_{p}$ aroung the values indicated above. We calculated

$$
\frac{d \sigma}{d t}=\frac{1}{16 \pi} \frac{1}{s^{2}}\left|T_{f i}\right|^{2}
$$

and the total cross section, using the optical theorem which reads for $s \gg m_{p}^{2}$ :

$$
\sigma_{T}(p p)=\frac{1}{s} \operatorname{Im}\left(T_{f i}\right)
$$

and compared to experiment. The experimental data on the total $p p$ and $p \bar{p}$ cross sections is very well described by the DL fit [2], [1]. We are only interested in the pomeron exchange part here. Therefore we take as "experimental" input the pomeron part of $\sigma_{T}(p p)$ in the DL parametrization [2]:

$$
\left.\sigma_{T}(p p)\right|_{\exp }=21.7\left(\frac{s}{\mathrm{GeV}^{2}}\right)^{0.0808} \mathrm{mb} .
$$

We imposed as constraint that our amplitude reproduced (41) exactly at $\sqrt{s}=23 \mathrm{GeV}$. 
With this procedure we found quite a satisfactory description of the data for $d \sigma / d t$ as shown in Fig. 5 for the following values of our parameters:

$$
\begin{gathered}
\rho=(435 \mathrm{MeV})^{2}, \\
a=0.32 \mathrm{fm}, \\
\kappa=0.74 \\
S_{p}\left(s=(23 \mathrm{GeV})^{2}\right)=0.87 \mathrm{fm} .
\end{gathered}
$$

From (38), (42), (43) we get

$$
\begin{aligned}
G_{2} & =(529 \mathrm{MeV})^{4}, \\
\kappa G_{2} a^{4} & =0.40 .
\end{aligned}
$$

Varying the parameters away from the values (42-45) did not lead to improvements. Also, the values (42, 45) are well within the range obtained in [21, 30] and quite compatible with the lattice results (91). In the following we will thus fix the vacuum parameters $\rho, a, \kappa$ to their values (42-44).

Now we consider $\sigma_{T}$ and $d \sigma / d t$ at higher energies $\sqrt{s}$, where we have then only one free parameter $S_{p}(s)$ left. We fix $S_{p}(s)$ again by requiring that our model reproduces the experimental value for the pomeron part of $\sigma_{T}(p p)$ according to (41).

On the other hand we follow [21], [27] and fit our calculated values of $\sigma_{T}(p p)$ from (32), (40) as shown in Fig. 14 to a power of $S_{p}$. We get a good description in the range

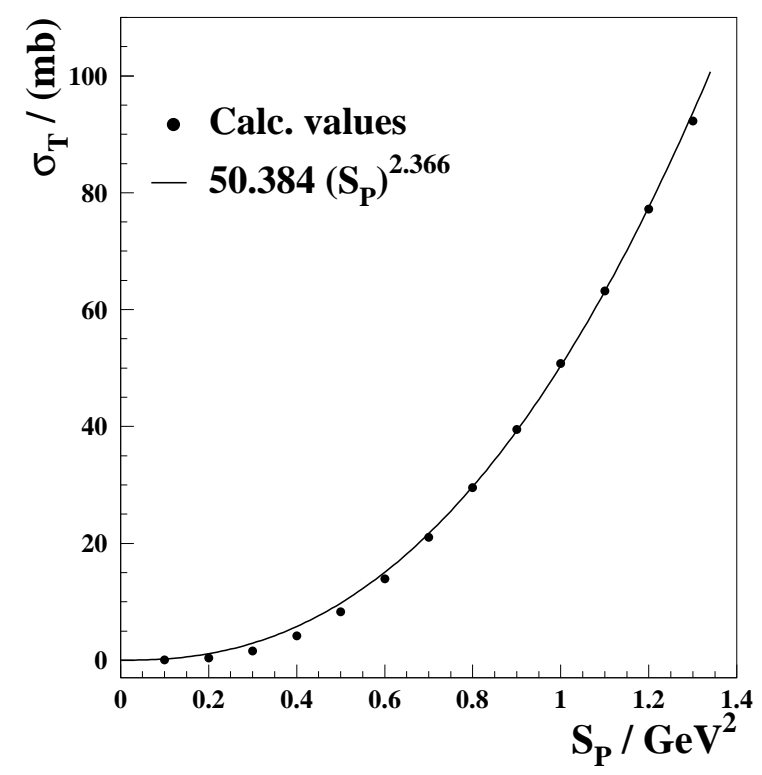

Figure 4: The total cross section $\sigma_{T}$ versus $S_{P}$. The solid points are the calculated values, the solid curve corresponds to the power fit.

$2.5 a \leq S_{p} \leq 4.0 a$ with

$$
\left.\sigma_{T}\left(S_{p}\right)\right|_{c a l}=50.384\left(S_{p}\right)^{2.366} \mathrm{mb}
$$


Equating (40) to (47) we get $S_{p}$ as function of $s$ :

$$
S_{p}(s)=0.700\left(\frac{s}{\mathrm{GeV}^{2}}\right)^{0.034} \mathrm{fm} .
$$

This leads to $S_{p}=0.86,0.87,0.93,1.07$ and $1.17 \mathrm{fm}$ for $\sqrt{s}=20,23,63,546$ and $1800 \mathrm{GeV}$, respectively. For comparison, the mean squared charge radius of the proton as
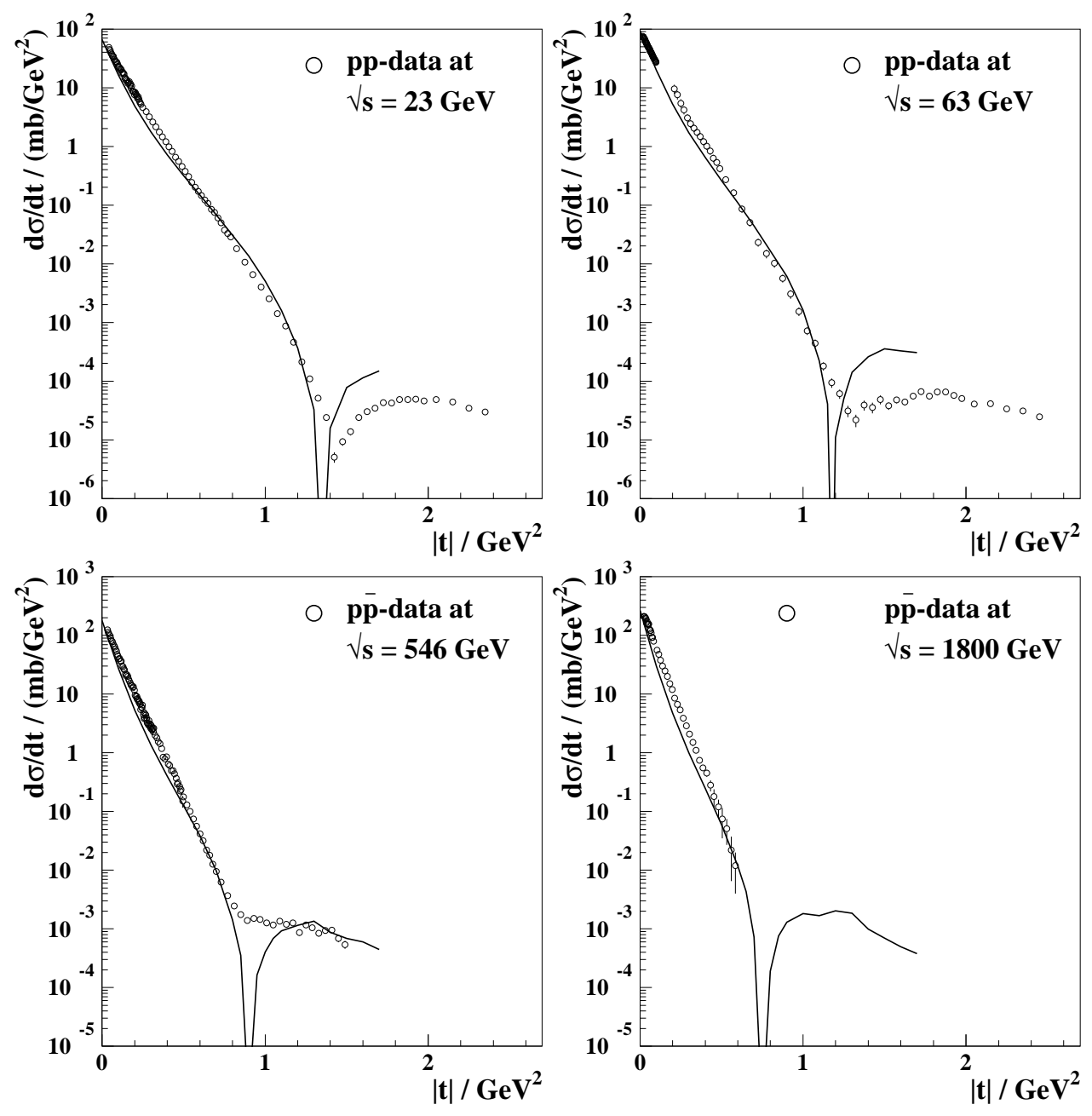

Figure 5: Differential elastic cross sections for c.m. energies $\sqrt{s}=23,63,546$ and 1800 $\mathrm{GeV}$. This corresponds to proton extension parameters $S_{P}=0.87,0.93,1.07$ and $1.17 \mathrm{fm}$. The data at $\sqrt{s}=23$ and $63 \mathrm{GeV}$ are from the ISR experiment [5]. The p $\bar{p}$ scattering data at $\sqrt{s}=546$ GeV are from the [6] and the data at $\sqrt{s}=1800$ GeV from [7].

determined from Lamb-shift measurements [45] is $r_{P}=0.89 \pm 0.014 \mathrm{fm}$. Now everything is fixed and we can compute the elastic scattering amplitude and $\sigma_{T}$ from (32). In Fig. 5 we present our results. A first observation is that for all energies the calculated differential distributions follow the experimental data quite well over many orders of magnitude. The 
fact that this is true up to $\sqrt{s}=1800 \mathrm{GeV}$ supports the description of the s-dependence by a $s$-dependent extension parameter $S_{p}(s)$.

For small $|t| \simeq 0.25 \mathrm{GeV}^{2}$ we get a change of slope (Fig. 6). Splitting the integrand

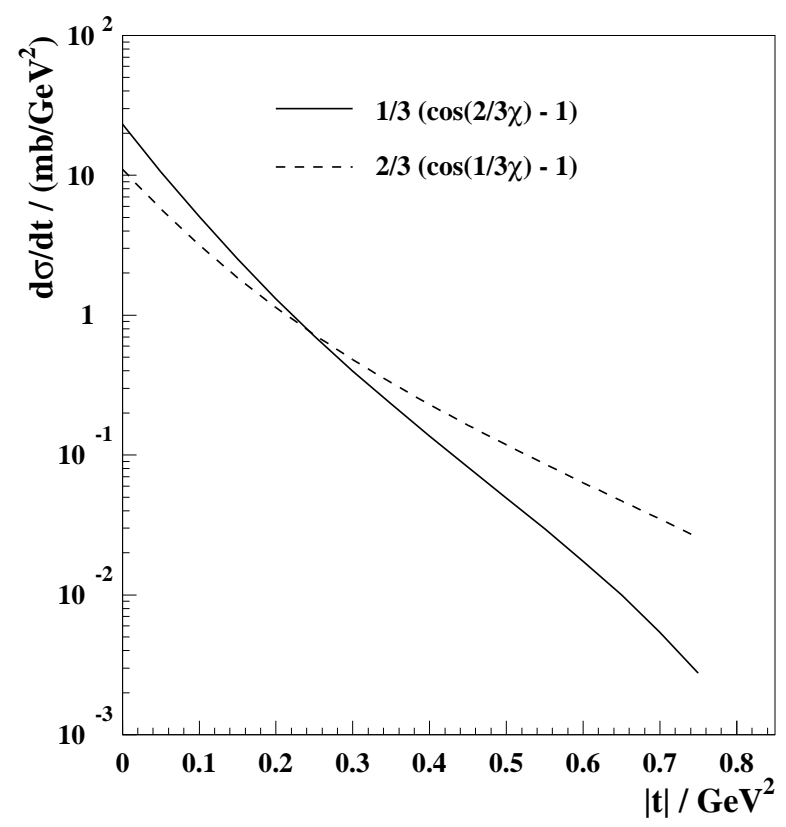

Figure 6: Differential elastic cross sections calculated for the values of $G_{2}=(529 \mathrm{MeV})^{4}$, $a=0.32 \mathrm{fm}$ and $S_{p}=0.87 \mathrm{fm}$ using as integrand of the amplitude (32) $\frac{1}{3}\left[\cos \left(\frac{2}{3} \chi\right)-1\right]$ (solid line) and $\frac{2}{3}\left[\cos \left(\frac{1}{3} \chi\right)-1\right]$ (dashed line) respectively.

of (32) into two contributions

$$
\frac{2}{3} \cos \left(\frac{1}{3} \chi\right)+\frac{1}{3} \cos \left(\frac{2}{3} \chi\right)-1=\frac{1}{3}\left[\cos \left(\frac{2}{3} \chi\right)-1\right]+\frac{2}{3}\left[\cos \left(\frac{1}{3} \chi\right)-1\right]
$$

we find that for $|t| \lesssim 0.25 \mathrm{GeV}$ the first term dominates and for $|t| \gtrsim 0.25 \mathrm{GeV}$ the second one dominates. Such a change of slope is indeed reported by experiments [38].

For all energies the imaginary part of our amplitude changes sign at some $t<0$. Due to the absence of a real part in (32) the calculated differential cross sections have a zero there. This causes an infinitely deep dip in our $t$-distributions. We expect this dip to be at least partly filled up once we change to more general quark configurations and include higher cumulant terms. The point at which the zero occurs in our calculation moves to smaller values of $|t|$ with increasing energy and is always in the region where experiments see a marked structure: At lower energies there is a dip in $p p$, and a shoulder in $p \bar{p}$ scattering, respectively. At the highest energies only $p \bar{p}$ data is available and one finds a shoulder. Thus our model produces structure in the $t$-distributions at the right place. But we should insert the warning that these dips occur at $|t| \simeq 1 \mathrm{GeV}^{2}$ where one would expect also perturbative effects to play a significant role. (cf. [40], [41]).

Of course, our model does not give a perfect fit to the data. At all energies our calculated curves are somewhat too steep at very small $|t|$. Our amplitude is purely 
imaginary and thus does not satisfy the relation between the phase and the $s$-dependence required by analyticity and Regge theory [3]. Also our $d \sigma / d t$ is the same for $p p$ and $p \bar{p}$ scattering, whereas experimentally these differ markedly in the dip region. This was nicely explained theoretically in 441] as an interference of single and double pomeron and three-gluon exchange. We will have to see if higher cumulant terms and/or a departure from the strict quark-diquark picture of the proton will lead us to an improvement on these points in our model.

In Fig. 7 we show our prediction for $d \sigma / d t$ in $p p$ scattering at $\sqrt{s}=14 \mathrm{TeV}$ corresponding to the LHC energy. The total cross section is again assumed to be given by the

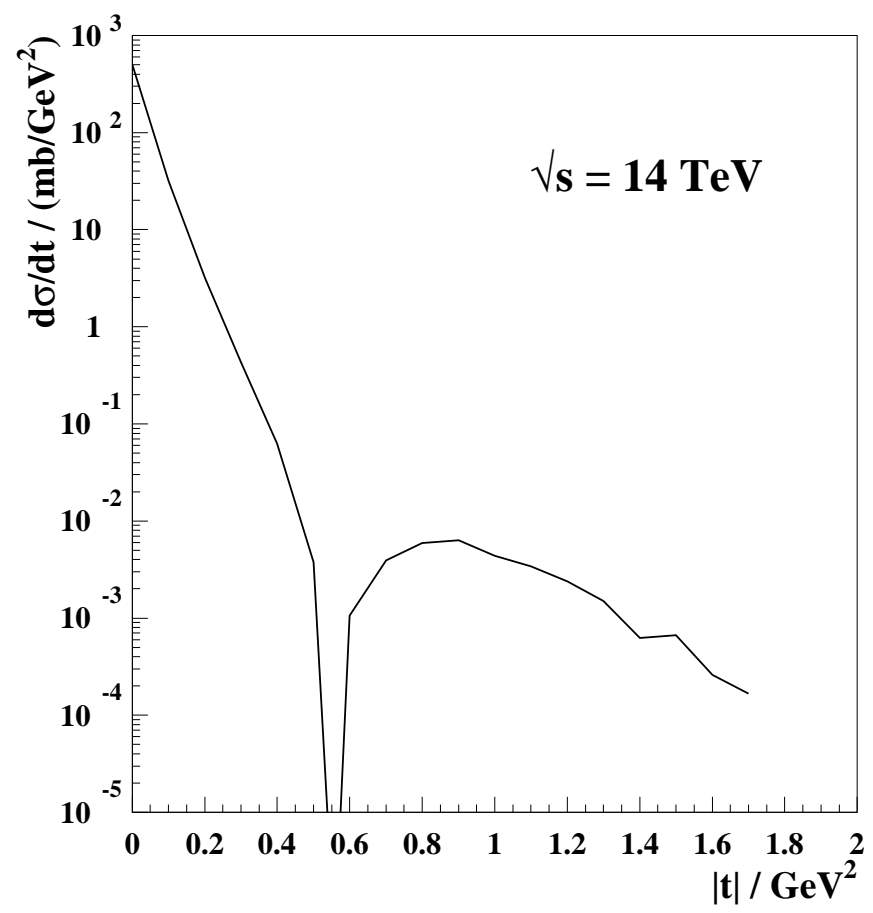

Figure 7: Prediction of the differential elastic cross section for the LHC energie $\sqrt{s}=$ $14 \mathrm{TeV}$.

DL parameterisation (41). This was used as constraint to fix the extension parameter $S_{p}$ according to Fig. 4 with the result

$$
S_{p}\left(s=(14 \mathrm{TeV})^{2}\right)=1.34 \mathrm{fm} .
$$

But the shape of $d \sigma / d t$ is a prediction of our model and we are looking forward to the corresponding experimental data.

We will show next that our results for $d \sigma / d t$ depend crucially on the string tension $\rho \neq 0$, i.e. on the confinement features of QCD. For this we plot in Fig. $8 d \sigma / d t$ for $\sqrt{s}=23 \mathrm{GeV}$ calculated for the same values of $G_{2}=(529 \mathrm{MeV})^{4}, a=0.32 \mathrm{fm}$ and $S_{p}=0.87 \mathrm{fm}$, but for different values of $\kappa$. For the purely non-abelian case $\kappa=1$ the fall of $d \sigma / d t$ is too steep, for the purely abelian, the non-confining case $\kappa=0$, the fall is much 

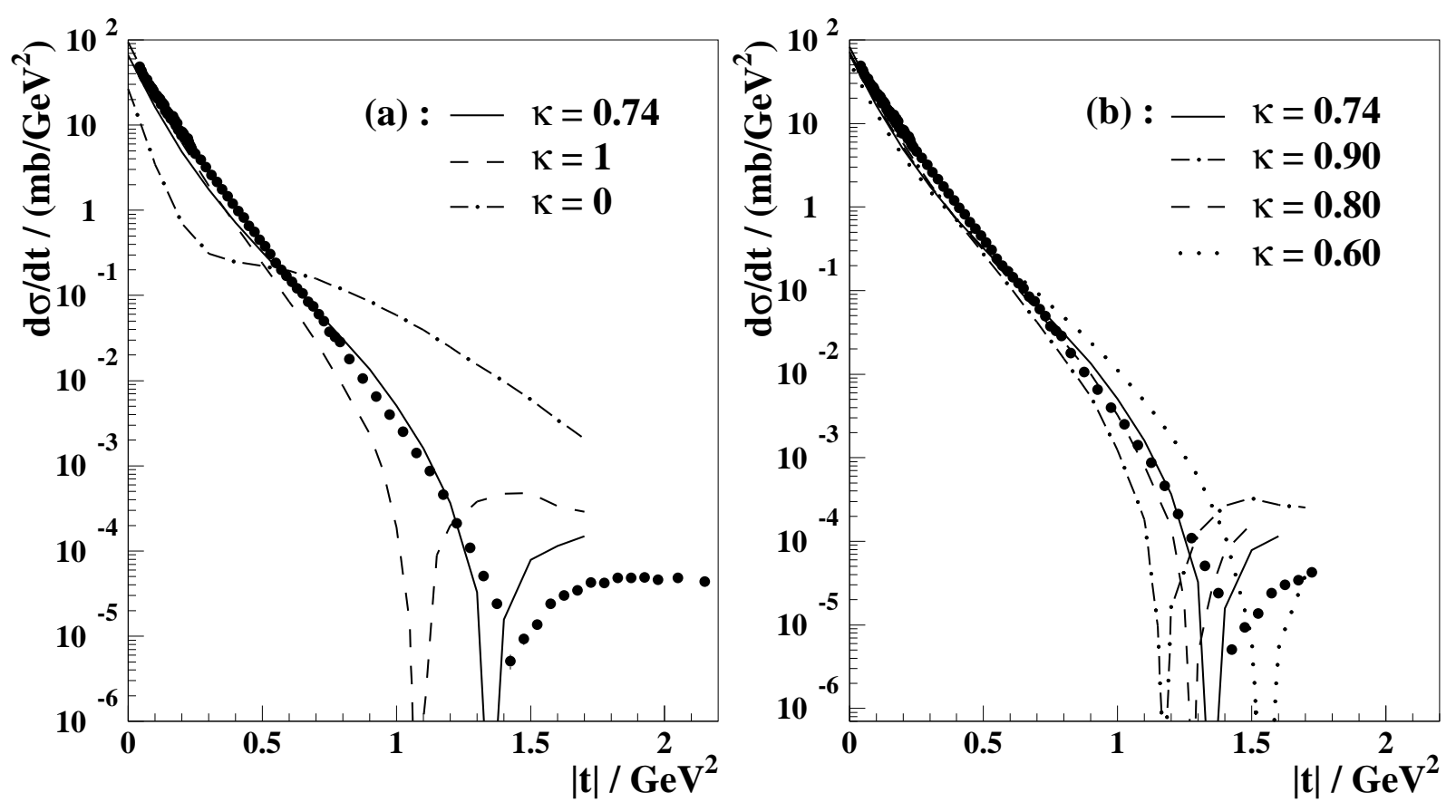

Figure 8: The variation of the differential elastic cross section calculated for the same values of $G_{2}=(529 \mathrm{MeV})^{4}, a=0.32 \mathrm{fm}$ and $S_{p}=0.87 \mathrm{fm}$; (a) for $\kappa=0.74$ (solid line), $\kappa=1$ (dashed line) and $\kappa=0$ (dash-dotted line); (b) for $\kappa=0.74$ (solid line), $\kappa=0.90$ (dash-dotted line), $\kappa=0.80$ (dashed line) and $\kappa=0.60$ (dotted line) together with the experimental data (solid points) at $\sqrt{s}=23 \mathrm{GeV}$. From (b) one can see, that a value of $\kappa$ around 0.74 is favoured by the data.

too slow with increasing $|t|$. The correct fall and dip position is obtained for $\kappa=0.74$, not far from the value determined from the lattice calculations (9).

To explain this we plot the profile function $\hat{J}_{M, M}(b)$ multiplied with $b$ for $\kappa=0$ and $\kappa=1$ versus $b$ in Fig. 9. As we can see, the two curves differ noticeably. For $\kappa=1$ we get a single maxima whereas for $\kappa=0$ we get two maxima. This can be understood by looking at the dependence of $\chi$ on $b$ for fixed transverse vectors $\boldsymbol{x}_{T}$ and $\boldsymbol{y}_{T}$ in the two cases. As we see from (23) $\chi$ is a sum of four terms (replace here $\bar{q}$ by the diquark $q q$ ) corresponding to $q-q, q-q q$ etc. interactions. For $\kappa=0$ the function $I\left(\boldsymbol{r}_{x}, \boldsymbol{r}_{y}\right)$ in (24) depends only on the difference $\boldsymbol{r}_{y}-\boldsymbol{r}_{x}$ of the parton positions, but for $\kappa=1 I\left(\boldsymbol{r}_{x}, \boldsymbol{r}_{y}\right)$ gets contributions from all "strings" spanned between $o$ and the $q$ 's and $q q$ 's positions ( Fig. 10). We get large contributions to $I$ if the arguments of the Bessel functions $K_{2,3}$ in (24) are small. For $\kappa=0$ this means that the partons $(q, q q)$ have to be close in transverse space, but for $\kappa=1$ it is only required that one parton be close to the string of the other parton. To see the consequences of this, we take as an example $\left|\boldsymbol{x}_{T}\right|=\left|\boldsymbol{y}_{T}\right|=4$ and $\boldsymbol{x}_{T}, \boldsymbol{y}_{T}, \boldsymbol{b}_{T}$ nearly parallel to each other (Fig. 10). For $\kappa=1$ we have the following situation. For small and medium values of $b$ the terms $I$ corresponding to the $q_{x}-q_{y}$ and $q q_{x}-q q_{y}$ interaction dominate in the sum for $\chi$ in (23). These functions $I$ decrease 


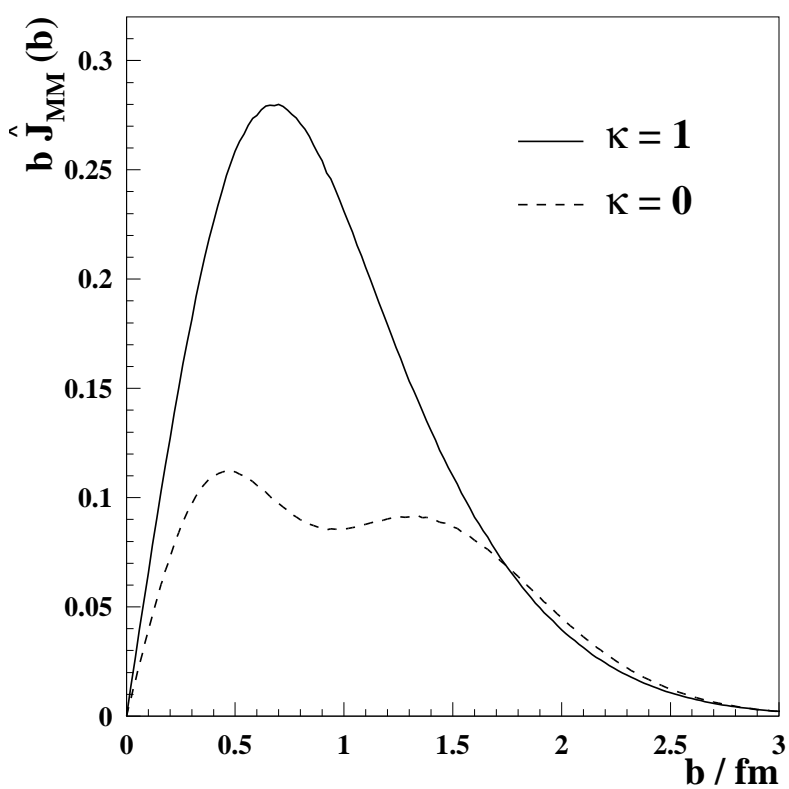

Figure 9: The dimensionless profile function $\hat{J}_{M M}(b)$ times $b$ versus $b$ for $G_{2}=$ $(529 \mathrm{MeV})^{4}, a=0.32 \mathrm{fm}$ and $S_{p}=0.87 \mathrm{fm}$ in the cases $\kappa=1$ (solid line) and $\kappa=0$ (dashed line).

with increasing $b$ nearly monotonously corresponding to $q_{x}$ moving away from the string $o-q_{y}$ and $q q_{y}$ away from the string of $o-q q_{x}$. But the interaction of $q_{y}$ with the string $o-q_{x}$ and $q q_{x}$ with the string $o-q q_{y}$ remain dominant. Compared to them the terms $I$ corresponding to the $q q_{x}-q_{y}$ which enters with negative sign in (23) stays smaller and also the function $\chi$ decreases nearly monotonously with increasing $b$. In summary: for $\kappa=1$ the function $\chi$ is sizable and practically always of the same sign as long as partons are close to strings of other partons, i.e. as long as projection of the two loops overlap. This picture, smeared out due to the integrations over the transverse vectors, is reflected in the shape of $\left(b \hat{J}_{M, M}(b)\right)$ for $\kappa=1$.

For $\kappa=0$ the function $\chi$ has a maximum at $b=0$, where the quarks and the diquarks of the two protons are closest to each other in transverse space. Then $\chi$ decreases rapidly for increasing $b$ on a scale given by the correlation length $a$, passes zero at some value $b_{0}$ and approaches a minimum. The latter corresponds to the situation where the quark of one proton is very close to the diquark of the other proton. Since there are no strings in this case all interactions except the one between $q q_{x}$ and $q_{y}$ are then negligeable and the latter enters with opposite sign to the $q_{x}, q_{y}$ and $q q_{x}, q q_{y}$ interaction in (23). For $b$ increasing further the transverse distance between all partons of the dipoles increase and $\chi$ goes to zero. At $b_{0}$ the expression in square brackets in (32) vanishes. Thus we expect - after smearing out through the integrations - to see a minimum in $b \hat{J}_{M M}(b)$ around $b_{0}$ and this is indeed seen in Fig. 9. Thus, through this sign change of $\chi$ we understand the two maxima in the shape of $b \hat{J}_{M, M}(b)$ for $\kappa=0$, and also that for $\kappa=0$ the first maximum in $b \hat{J}_{M M}(b)$ occurs for a smaller value of $b$ than for $\kappa=1$. After the 
(a):
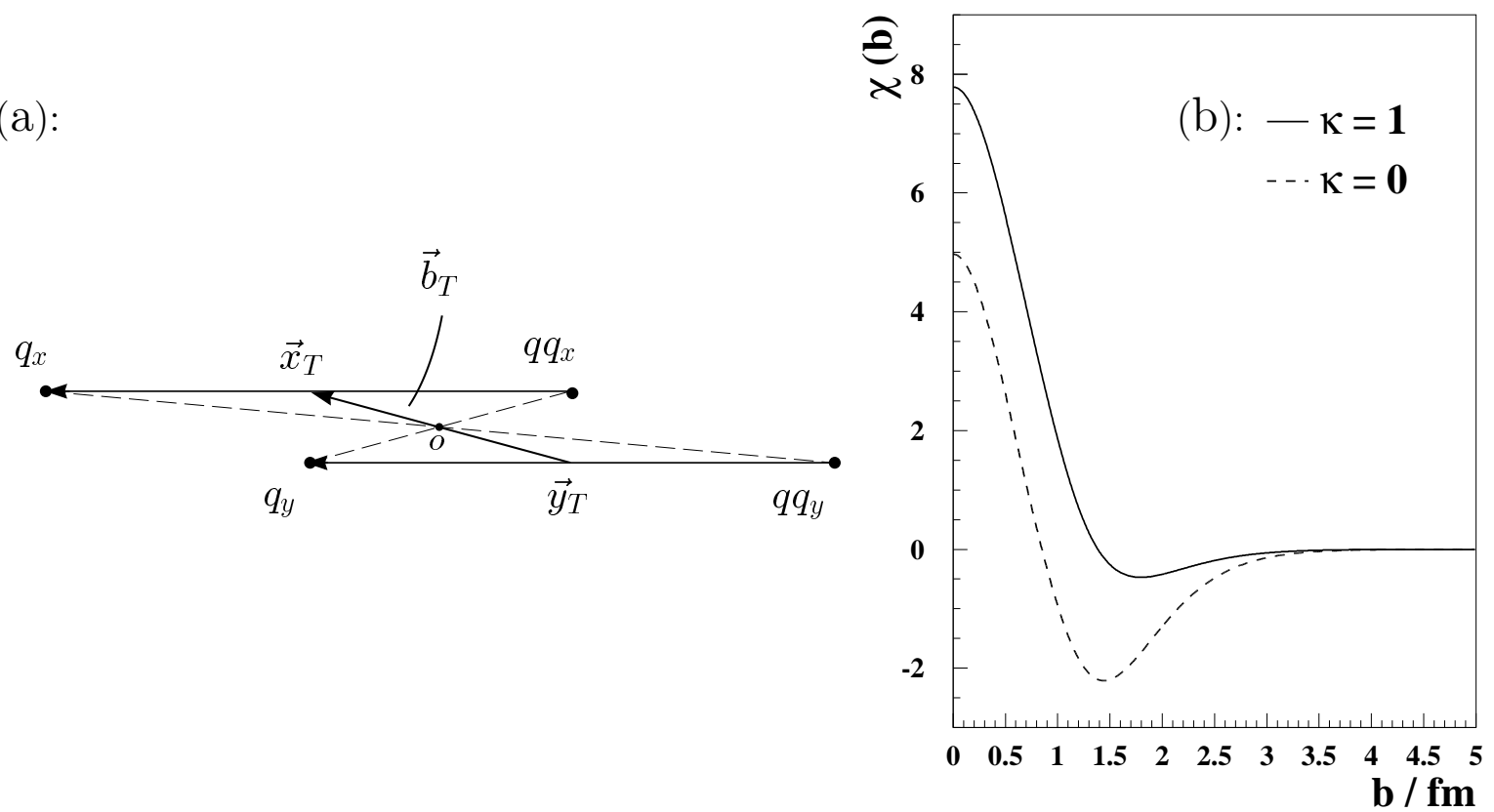

Figure 10: (a) The transverse positions of the quarks $q_{x}, q_{y}$ and diquarks $q q_{x}, q q_{y}$ for parallel loops. The dashed lines represent the "strings" spanned between o and the q's and qq's positions. (b) The correlation function $\chi(b)$ versus $b$ for $\left|\boldsymbol{x}_{T}\right|=\left|\boldsymbol{y}_{T}\right|=4 a$ and $\boldsymbol{x}_{T}, \boldsymbol{y}_{T}, \boldsymbol{b}_{T}$ parallel to each other in the cases $\kappa=1$ (solid line) and $\kappa=0$ (dashed line). The curves correspond to loop configurations sketched in (a), namely to parallel loops lying on each other for $b=0$ and moving for increasing $b$ in $+\boldsymbol{x}_{T}$ direction and $-\boldsymbol{y}_{T}$ direction respectively.

Fourier-Bessel transformation in (32) this translates immediately in the slower decrease of $d \sigma / d t$ for $\kappa=0$ compared to $\kappa=1$. The dip in $d \sigma / d t$ is generated by a cancellation of positive and negative contributions from $b \hat{J}_{M M}(b) J_{0}(b a \sqrt{|t|})$. For $\kappa=1$ and $\kappa=0.74$ the function $b \hat{J}_{M M}(b)$ is "smooth" and the oscillating Bessel function brings the integral (32) "easily" to zero for some $|t|$. For $\kappa=0$ the "oscillation" of $b \hat{J}_{M M}(b)$ together with the oscillation of $J_{0}(b a \sqrt{|t|})$ can produce a dip only at much higher $|t|$. From this point of view the structure of the $p p$ scattering amplitude gives us direct information that a string formation between quarks in QCD is essential also here.

We can now also understand how the shrinkage of our calculated differential cross sections (see Fig. 5) for increasing c.m. energies is caused: We found above for $\kappa=0.74$ that the shape of $d \sigma / d t$ is controlled by string scattering. We assumed $S_{p}$ to increase with $s$ which means increasing lengths of the string sizes and thus still overlap for larger values of the impact parameter $b$. This in turn translates into steeper forward peaks of the scattering amplitude and so into steeper elastic differential cross sections at higher energies. 

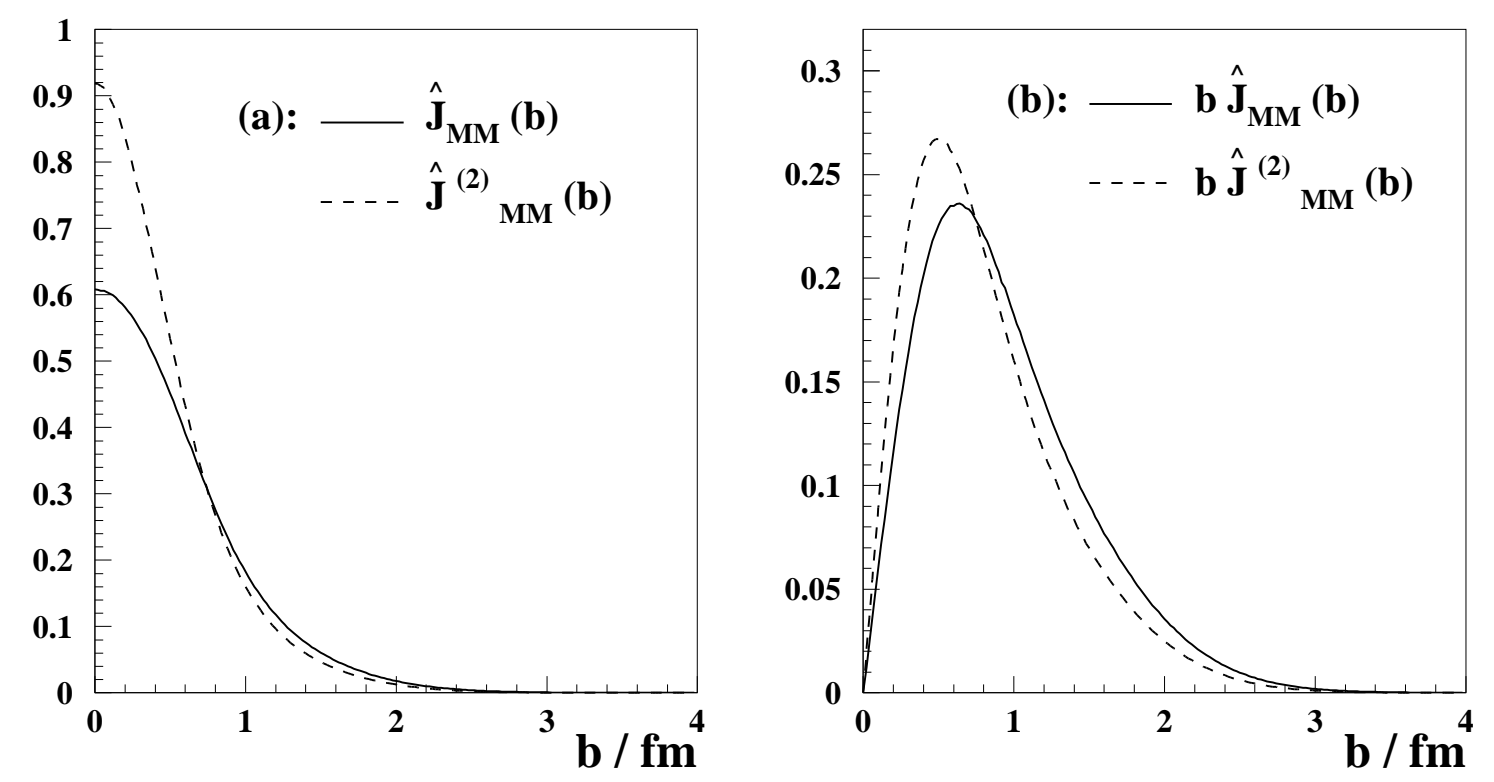

Figure 11:

For $\sqrt{s}=23 \mathrm{GeV}$ (a) the profile functions $\hat{J}_{M M}(b)$ (solid line) and $\hat{J}_{M M}^{(2)}(b)$ (dashed line) as a function of $b$. (b) The profile functions $\hat{J}_{M M}(b)$ (solid line) and $\hat{J}_{M M}^{(2)}(b)$ (dashed line) multiplied with $b$ versus $b$.

In Fig. 11 we compare our profile function $\hat{J}_{M M}(b)$ from (32) with the corresponding one, $J_{M M}^{(2)}(b)$ of (30), where only the term of order $\chi^{2}$ is kept. We see from Fig. 10 that this can only be a good approximation for larger values of $b$ and indeed, the two functions $\hat{J}_{M M}$ and $\hat{J}_{M M}^{(2)}$ are quite similar for $b \gtrsim 2 a$, but are rather different for $b \lesssim 2 a$. But for the total cross section and the slope parameter at $t=0$ which are obtained from integrals over $b \hat{J}_{M M}(b)$ and $b^{2} \hat{J}_{M M}(b)$ this does not make much difference. On the other hand at larger and larger $|t|$ the integrals with the Bessel function in (32), (30) probes smaller and smaller vales of $b$ in $\hat{J}_{M M}(b)$ and then the differences between $\hat{J}_{M M}$ and $\hat{J}_{M M}^{(2)}$ show up clearly.

Finally we come back to the partial wave expansion for the $\mathrm{T}$ matrix element at high energies (35) and discuss the case of total absorption which means $\eta_{l}=0$ for the inelasticities $\eta_{l}$ in (36). Thus total absorption requires $\left|\hat{J}_{M M}(b)\right| \leq 1$ which is again satisfied for our amplitude (32). At $\sqrt{s}=23 \mathrm{GeV}$ this can be read off from Fig. 11a.

\section{Meson-meson and meson-baryon scattering}

\subsection{Meson-meson scattering}

Meson-meson scattering is the reaction best suited to apply our formula (32). Unfortunately there is no data available for small $t$ elastic meson-meson-scattering at sufficiently 


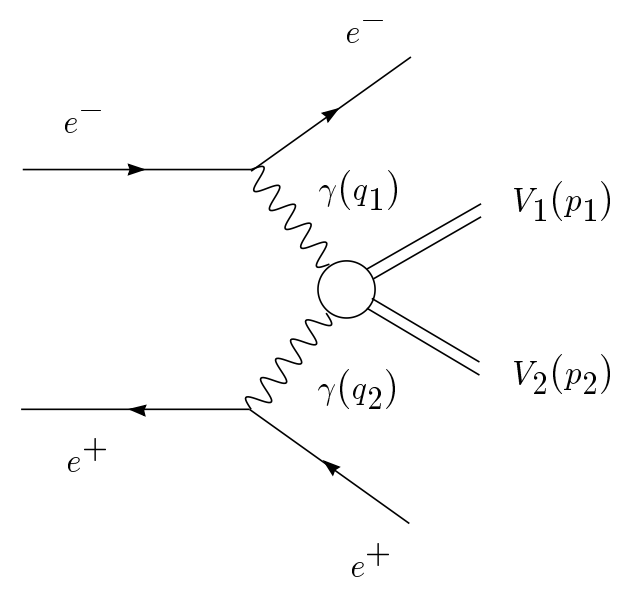

Figure 12: Vector meson production in two photon processes at $e^{+} e^{-}$colliders.

large c.m. energies. But the reaction

$$
\gamma\left(q_{1}\right)+\gamma\left(q_{2}\right) \rightarrow V_{1}\left(p_{3}\right)+V_{2}\left(p_{4}\right), \quad V_{1,2}=\rho, \omega, \phi
$$

is nearly as good to extract mesonic differential cross sections as a purely mesonic one and it can be studied for instance at LEP [42] in photon-photon processes (Fig. 12). Certainly at least part of the reaction (51) is due to the vector dominance model 443 mechanism: The photons fluctuate before the collision into vector mesons which then interact. The hadronic interaction can be calculated in the way described in Sect. 3. Thus in essence we can regard the reaction (51) as elastic scattering of vector mesons. This approximation should be best for quasi real photons in (51), i.e. for very small virtualities $\left|q_{1}^{2}\right|$ and $\left|q_{2}^{2}\right|$.

In this spirit we discuss now $\rho \rho$ elastic scattering as an example. In our amplitude (32) we have already fixed the vacuum parameters $\rho, \kappa$ and $a$ in Sect. 4 , but we still have to choose the $\rho$ extension parameter $S_{\rho}$ and we assume it to be equal to the $\pi$ extension parameter as determined in the next section

Indeed, data on $\rho$ - $N$ total cross sections from photoproduction on nuclei at low energies [44] show $\sigma_{T}(\rho N) \approx \sigma_{T}(\pi N)$. Thus we assume:

$$
S_{\rho}=0.60 \mathrm{fm} \text { for } \sqrt{s}=20 \mathrm{GeV} .
$$

Now everything is fixed and we can calculate $d \sigma / d t$ for $\rho$ - $\rho$ scattering.

In Fig. 13 we plot $d \sigma / d t$ normalised to 1 at $t=0$. Assuming the vector dominance model and neglecting contributions from non-diagonal scattering $\omega \omega \rightarrow \rho \rho$ etc., Fig. 13 also gives the shape of $d \sigma / d t$ for $\gamma \gamma \rightarrow \rho \rho$-scattering. It would be very interesting to have data for instance from LEP2 to compare with our $t$-distribution.

\subsection{Differential cross sections for meson-proton scattering}

Here we calculate elastic differential cross sections for $\pi^{ \pm} p$ and $K^{ \pm} p$ scattering at $\sqrt{s}=$ $19.5 \mathrm{GeV}$. This is the largest energy for which data for these reactions exist. 


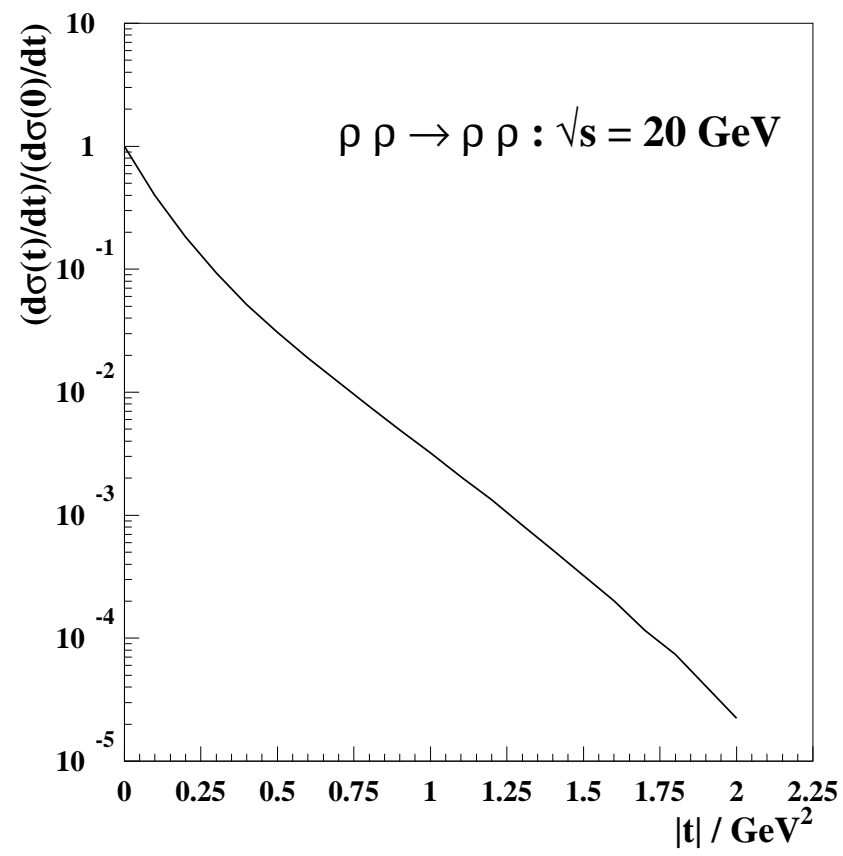

Figure 13: Prediction for the differential elastic cross section of $\rho \rho$-scattering at $\sqrt{s}=20$ $\mathrm{GeV}$ using $S_{\rho}=0.60 \mathrm{fm}$.

In our scattering amplitude (32) we have already fixed vacuum parameters $\rho, \kappa, a$ (4244). We consider the proton as a quark-diquark system with extension parameter $S_{p}$ and use $S_{p}=0.86 \mathrm{fm}$ from (48). To fix the meson extension parameters we again normalise our total cross sections to the pomeron parts in the DL parametrisations of the $\pi^{ \pm} p$ and $K^{ \pm} p$ total cross sections [2].

$$
\begin{aligned}
& \left.\sigma\left(\pi^{ \pm} p\right)\right|_{\text {Pom. }}=22.0 \mathrm{mb} \\
& \left.\sigma\left(K^{ \pm} p\right)\right|_{\text {Pom. }}=19.1 \mathrm{mb} .
\end{aligned}
$$

This leads to

$$
\begin{aligned}
S_{\pi} & =0.60 \mathrm{fm}, \\
S_{K} & =0.55 \mathrm{fm} .
\end{aligned}
$$

For comparison the electromagnetic radii are [46]: $r_{\pi}=0.66 \pm 0.01 \mathrm{fm}, r_{K}=0.58 \pm 0.04 \mathrm{fm}$. Fig. 14 shows our results for the $\pi^{ \pm} p$ and $K^{ \pm} p$ elastic differential cross sections. As we can see the experimental data are again reproduced quite well. As in $p p$ scattering the slopes of the calculated cross sections are somewhat too large for very small $|t|$. Furthermore all our $t$-distributions are slightly below the experimental data up to $|t| \simeq 0.5 \mathrm{GeV}$. This could again be due to with the missing real part in the amplitude (32), a problem which might be cured once we go beyond the second cumulant approximation.

We also note that the c.m. energy $\sqrt{s}=19.5 \mathrm{GeV}$ considered here is not very high and - in Regge language - effects of nonleading trajectories are still sizable and more so 

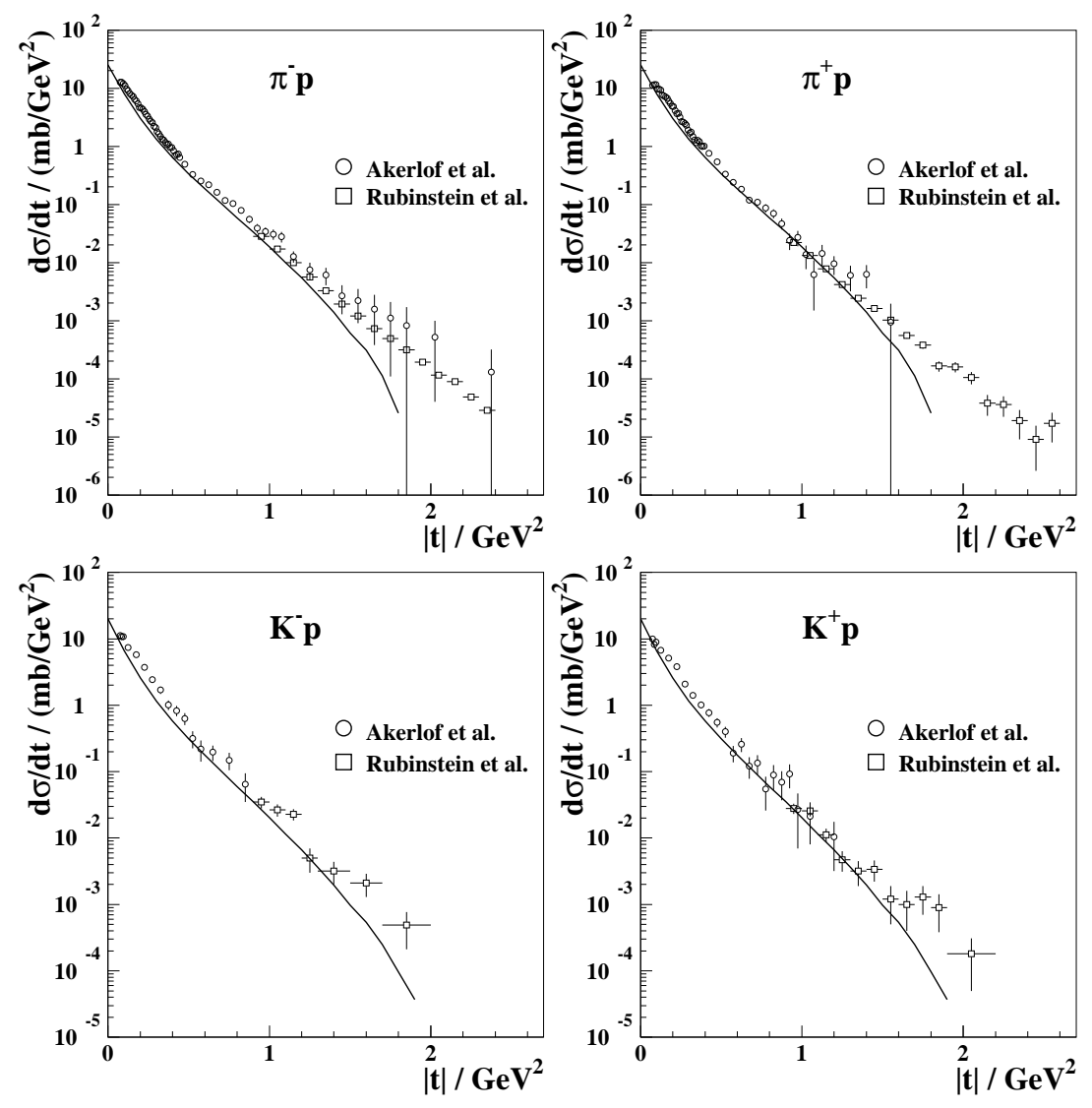

Figure 14: Differential elastic cross sections for $\pi^{ \pm} p$ and $K^{ \pm} p$ scattering at $\sqrt{s}=19.5$ GeV. This corresponds to $S_{P}=0.86 \mathrm{fm}, S_{\pi}=0.60 \mathrm{fm}$ and $S_{K}=0.55 \mathrm{fm}$. The data are from 8 .

for $\pi^{-} p, K^{-} p$ than for $\pi^{+} p, K^{+} p$ scattering. Our calculation does not contain any nonleading Regge-exchanges and thus should agree better with $\pi^{+} p, K^{+} p$ than $\pi^{-} p, K^{-} p$ scattering. This is not incompatible with the results shown in Fig. 14 .

Due to the smaller extension parameters of the mesons our calculated $t$-distributions for meson-proton scattering are flatter than those for proton-proton scattering. Also our calculation gives dips only around $|t| \approx 2 \mathrm{GeV}^{2}$ and we would not believe our model to be reliable at such high $|t|$-values.

The squares of the ratios of our extension parameters of mesons and the proton are

$$
\left(\frac{S_{\pi}}{S_{p}}\right)^{2}=0.49,\left(\frac{S_{K}}{S_{p}}\right)^{2}=0.41,\left(\frac{S_{K}}{S_{\pi}}\right)^{2}=0.84
$$

The corresponding ratios for the mean squared electromagnetic radii are [45, 46]

$$
\frac{r_{\pi}^{2}}{r_{p}^{2}}=0.55, \frac{r_{K}^{2}}{r_{p}^{2}}=0.42, \frac{r_{K}^{2}}{r_{\pi}^{2}}=0.77
$$


The ratios (55) follow the trends of (56) but certainly are not equal to them. It is particularly noteworthy that in our model the facts that (i) the $K^{ \pm} p$ total cross sections are smaller than the $\pi^{ \pm} p$ ones and (ii) the $t$ distributions for $K^{ \pm} p$ flatter than for $\pi^{ \pm} p$ are both reproduced quantitatively by a smaller extension parameter $S_{K}$ compared to $S_{\pi}$. In the additive quark model [4] on the other hand one has to assume a different cross section for the scattering of $u, d$ on $u, d$ and $u, d$ on $s$ quarks which is hard to understand since the gluon interaction is flavour-blind.

\section{Conclusion}

In this article we have presented calculations of amplitudes for elastic proton-proton, meson-meson, and meson-proton scattering at high energies and small momentum transfer. Our model is based on functional integral techniques [19] and an appropriate evaluation of such integrals in the framework of the stochastic vacuum model [22, 21] In comparison with previous work [21, 23, 27] we have now made a cumulant expansion for the correlation function of two Wegner-Wilson loops instead of an expansion in terms of the number of field strength correlators. We found that this latter expansion can only be justified for medium and large impact parameters, but gives, nevertheless, reasonable results for the total cross section and the slope parameter at $t=0$.

As parameters in our model we have the QCD vacuum parameters: the gluon condensate $G_{2}$, the non-abelian parameter $\kappa$, and the correlation length $a$. In addition we have the $s$-dependent hadron extension parameters $S_{H}(s)$.

We fixed the $S_{H}(s)$ by requiring the total cross sections to agree with the experimental values. In this way we found quite a good description of $d \sigma / d t$ for $p p, p \bar{p}, \pi^{ \pm} p$ and $K^{ \pm} p$ scattering for vacuum parameters $G_{2}, \kappa, a$ or equivalently the string tension $\rho, \kappa$ and $a$ as given in (42-44). These values are close to the corresponding values obtained from lattice data [32, 33], from previous investigations of high energy scattering [21, 30] and from various other low energy phenomena (see [31] for a review). The parameters $S_{H}(s)$ came out close to the known electromagnetic radii of the hadrons for energies $\sqrt{s} \approx 20 \mathrm{GeV}$ and were required to increase slowly with $s$ according to (48).

As in previous work 21] the fact that $\sigma_{T}(K p)$ is smaller than $\sigma_{T}(\pi p)$ is related in our model to a smaller extension parameter $S_{K}$ compared to $S_{\pi}$.

The dip structure in $p p$ scattering around $|t| \approx 1.5 \mathrm{GeV}^{2}$ was seen to depend crucially on the non-abelian character of the gluon field strength correlator, i.e. on $\kappa \neq 0$. This leads to the string formation [22] and the area law for the Wegner-Wilson loop with static quarks. In our model these strings play again a crucial role in high energy scattering in producing the correct $t$-distributions.

At very small values of $|t|$ our model gives a change of slope related to the symmetric and antisymmetric combinations of the multi-gluon exchange as explained after (25).

As stated above one of the main ingredients of our model is a cumulant expansion for the correlation function of 2 Wegner-Wilson loops which we truncated after the second cumulant. But we can easily see that the main features of our model will remain unchanged 
if the matrix cumulant expansion (19) converges. Then the complete sum of cumulants in (19) is a $9 \times 9$ matrix, invariant under $S U(3)$ rotations. We will again obtain a decomposition for it as for $C_{2}$ in (23) ff in terms of the projectors $P_{s}$ and $P_{a}$ with invariant functions $\chi_{s, a}$ multiplying them etc. Of course the detailed shape of these functions will be different.

Going back to our amplitude (32), we can expand the cosines in powers of $\chi$. Maybe the $\chi^{2}$-term - as kept in the work [21] - could be interpreted as exchange of two nonperturbative gluons, as single bare pomeron exchange, the $\chi^{4}$-term as exchange of four nonperturbative gluons, as double pomeron exchange, etc. At the moment, however, such an identification is purely speculative.

In all this work we have treated baryons as quark-diquark systems, i.e. we assumed 2 quarks of the 3 valence quarks of a baryon to be close together in transverse directions. This picture is supported e.g. by the investigation of [28 where it is shown to give an explanation for the apparent absence of odderon couplings of the proton at small

$|t|$. In future work we plan to investigate meson-baryon and baryon-baryon scattering treating baryons as 3-quark systems with arbitrary distances between the quarks. Also the dependence of the results on the surfaces spanned into the loops (see Fig. 2) in order to apply the non-abelian Stokes theorem will be investigated.

To summarise: We have presented a model where the $t$-distributions of elastic hadronhadron scattering are related quantitatively to the parameters of the vacuum in nonperturbative QCD. The total cross sections which rise with energy were used to fix the energy-dependent effective strong interaction extension parameters of the hadrons. A theoretical calculation of these extension parameters remains a challenge.

\section{Acknowledgements}

We are grateful to H. G. Dosch, A. Donnachie, E. Ferreira, A. Hebecker, W. Kilian, P. V. Landshoff, M. Rüter, and Yu. A. Simonov for many fruitful discussions. Special thanks are due to E. Meggiolaro for clarifying discussions concerning the lattice data and for extracting from the latter the vacuum parameters in the form suitable for us. 


\section{References}

[1] R. M. Barnett et al. (Particle Data Group), Eur. Phys. J. C (1998)

[2] A. Donnachie, P. V. Landshoff, Phys. Lett. B 296 (1992) 227.

[3] P. D. B. Collins: "An Introduction to Regge Theory" , Cambridge University Press, Cambridge, U.K. 1977; L. Caneschi, ed.: "Regge Theory of low $p_{T}$ hadronic interaction", North Holland, Amsterdam 1989.

[4] E. M. Levin and L. L. Frankfurt, JETP Lett. 2 (1965) 65; H. J. Lipkin and F. Scheck, Phys. Rev. Lett. 16 (1966) 71; H. J. Lipkin, Phys. Rev. Lett. 16 (1966) 1015; J. J. J. Kokkedee and L. van Hove, Nuovo Cimento A 42 (1966) 711.

[5] A. Böhm et al., Phys. Lett. 49B (1974) 491; E. Nagy et al., Nucl. Phys. B 150 (1979) 221; A. Breakstone et al., Nucl. Phys. B 248 (1984) 253; U. Amaldi et al., Phys. Lett. B 53 (1973) 231; U. Amaldi et al., Phys. Lett. B 62 (1976) 460; U. Amaldi, K. R. Schubert, Nucl. Phys. B 166 (1980) 301; N. Amos et al., Phys. Lett. B 120 (1983) 460.

[6] M. Bozzo et al., Phys. Lett.B 147 (1984) 385; UA4 Collaboration, D. Bernhard et al., Phys. Lett.B 198 (1987) 583.

[7] N. A. Amos et al.,Phys. Lett.B 243 (1990) 158; N. A. Amos et al.,Phys. Rev. Lett. 63 (1989) 2784.

[8] C. W. Akerlof et al., Phys. Rev. D 14 (1976) 2864; R. Rubinstein et al., Phys. Rev. D 30 (1984) 1413.

[9] A. Donnachie, P. V. Landshoff, Nucl. Phys. B 244 (1984) 322; Nucl. Phys. B 267 (1986) 690; Phys. Lett. B 185 (1987) 403.

[10] V. S. Fadin, E. A. Kuraev, L. N. Lipatov: Phys. Lett. B60, (1975) 50;

E. A. Kuraev, L. N. Lipatov, V. S. Fadin: Sov. Phys. J.E.T.P. 44, (1976) 443; 45, (1977) 199;

L. N. Lipatov: Sov. J. Nucl. Phys. 23, (1976) 338;

Ya. Ya. Balitskii, L. N. Lipatov: Sov. J. Nucl. Phys. 28, (1978) 822;

L. N. Lipatov: Sov. Phys. J.E.T.P. 63, (1986) 904;

L. N. Lipatov: "Pomeron in Quantum Chromodynamics", in "Perturbative Quantum Chromodynamics" (A. H. Mueller, Ed.), World Scientific, Singapore, 1989;

A. R. White: Int. J. Mod. Phys. A6, (1990) 1859; Nucl. Phys. B (Proc. Suppl.) 12 (1990) 190; Nucl. Phys. B 25 (1992) 167;

J. Bartels: Z. Phys. C60, (1993) 471.

[11] G. 't Hooft: Nucl. Phys. B72, 461 (1974);

G. Veneziano: Nucl. Phys. B74, 365 (1974); Phys. Lett. B52, 220 (1974); Nucl. 
Phys. B117, 519 (1976);

M. Ciafaloni, G. Marchesini, G. Veneziano: Nucl. Phys. B98, 472, 493 (1975);

A. Capella, U. Sukhatme, Chung-I Tan, J. Tran Thanh Van: Phys. Lett. B81, 68 (1979);

A. Capella, U. Sukhatme, J. Tran Thanh Van: Z. Phys. C3, 329 (1980);

B. Andersson, G. Gustafson, G. Ingelman, T. Sjöstrand: Phys. Rep. C97, 31 (1983);

X. Artru: Phys. Rep. C97, 147 (1983);

B. Andersson, G. Gustafson, B. Nilsson-Almqvist: Nucl. Phys. B281, 289 (1987);

K. Werner: Phys. Rep. C232, 87 (1993).

[12] R. C. Hwa: Phys. Rev. D22, 759, 1593 (1980);

R. C. Hwa, M. Sajjad Zahir: Phys. Rev. D23, 2539 (1981);

R. C. Hwa: "Central production and small angle elastic scattering in the valon model", Proc. 12th Int. Symp. Multiparticle Dynamics, Notre Dame, 1981 (W. D. Shephard, V. P. Kenny, eds.) World Scientific, Singapore 1982

[13] H. Cheng, T. T. Wu: "Expanding Protons", MIT Press, Cambridge, Mass. 1987, and references cited therein.

[14] T. T. Wu, C. N. Yang: Phys. Rev. B137, 708 (1965);

T. T. Chou, C. N. Yang: Phys. Rev. B170, 1591 (1968); Phys. Rev. D19, 3268 (1979); Phys. Lett. B128, 457 (1983); Phys. Lett. B244, 113 (1990);

J. Dias de Deus, P. Kroll: Phys. Lett. B60, 375 (1976); Nuovo Cimento A37, 67 (1977);

P. Kroll: Z. Phys. C15, 67 (1982);

J. Hüfner, B. Povh: Phys. Rev. D46, 990 (1992); J. Hüfner, B. Povh, E. Wälde: Z. Phys. C 63 (1994) 631.

[15] J. R. Forshaw and D. A. Ross, Quantum chromodynamics and the pomeron, Cambridge University Press (1997).

[16] P. V. Landshoff and O. Nachtmann, Z. Phys. C 35 (1987) 405.

[17] M. A. Shifman, A. I. Vainstein, and V. I. Zakharov, Nucl. Phys. B 147 (1979) 385, $448,519$.

[18] M. B. Voloshin: Nucl. Phys. B154 (1979) 365;

H. Leutwyler: Phys. Lett. B98 (1981) 447;

D. Gromes: Phys. Lett. B115 (1982) 482;

V. N. Baier, Yu. F. Pinelis: Phys. Lett. B116 (1982) 179;

O. Nachtmann, A. Reiter: Z. Phys. C24 (1984) 283.

[19] O. Nachtmann, Ann. Phys. 209 (1991) 436.

[20] A. Kraemer, H. G. Dosch, Phys. Lett. B 252 (1990) 669; B 272 (1991) 114; H. G. Dosch, E. Ferreira, A. Kraemer, Phys. Lett. B 289 (1992) 153; 
[21] H. G. Dosch, E. Ferreira, A. Krämer, Phys. Rev. D50 (1994) 1992.

[22] H. G. Dosch, Phys. Lett. B 190 (1987) 177; H. G. Dosch, Yu. A. Simonov, Phys. Lett. B 205 (1988) 339; Yu. A. Simonov, Nucl. Phys. B 307512.

[23] H. G. Dosch, T. Gousset, G. Kulzinger, H. J. Pirner, Phys. Rev. D55 (1997) 2602; H. G. Dosch, T. Gousset, H. J. Pirner, Phys. Rev. D57 (1998) 1666; G. Kulzinger, H. G. Dosch, H. J. Pirner: Diffractive Photo- and Leptoproduction of Vector Mesons $\rho, \rho^{\prime}$ and $\rho^{\prime \prime}$ hep-ph/9806352.

[24] W. Buchmueller, A. Hebecker, Nucl Phys. B476 (1996) 203;

W. Buchmueller, M. F. McDermott, A. Hebecker: Phys. Lett. B404 (1997) 353;

Phys. Lett. B410 (1997) 304;

W. Buchmueller, M. F. McDermott, A. Hebecker: Nucl Phys. B487 (1997) 283;

A. Hebecker: Nucl Phys. B505 (1997) 349.

[25] M. Derrik et al. (ZEUS Koll.): Phys. Lett. B315 (1993) 481; Z. Phys. C68 (1995) 569 ;

T. Ahmed et al. (H1 Koll.): Nucl Phys. B429 (1994) 477; Phys. Lett. B348 (1995) 681 ;

C. Adloff et al. (H1 Koll.): Z. Phys. C76 (1997) 613; Phys. Lett. B428 (1998) 206.

[26] O. Nachtmann: High Energy Collisions and Nonperturbative QCD, in "Perturbative and Nonperturbative Aspects of Quantum Field Theory", H. Latal, W. Schweiger (eds.), Springer Verlag, Berlin, Heidelberg (1997).

[27] H. G. Dosch, E. Ferreira: Phys. Lett. B318 (1993) 197;

E. Ferreira, F. Pereira: Phys. Rev. D55 (1997) 130; ibid. D56 (1997) 179;

M. Rüter, H. G. Dosch: Phys. Rev. D57 (1998) 4097.

[28] M. Rüter, H.G. Dosch, Phys. Lett. B 380 (1996) 177.

[29] M. Rüter, H. G. Dosch, O. Nachtmann: Qdd C-P contributions to diffractive processes, report hep-ph/9806342 (1998), submitted to Phys. Rev. D.

[30] M. Rüter, Quark-Confinement und diffraktive Hadron-Streuung im Modell des stochastischen Vakuums, PhD thesis, Univ. of Heidelberg (1997).

[31] H. G. Dosch, "Nonperturbative methods in Quantum Chromodynamics", Prog. in Part. and Nucl. Phys. 33 (1994) 121.

[32] A. DiGiacomo, H. Panagopoulos, Phys. Lett. B 285 (1992) 133; A. DiGiacomo, E. Meggiolaro, H. Panagopoulos, Nucl. Phys. B483 (1997) 371.

[33] E. Meggiolaro, Field Strength Correlators in QCD: New Fits to the Lattice Data, Univ. of Heidelberg report HD-THEP-98-34 (1998). 
[34] I. Ya. Aref'eva, Theor. Math. Phys. 43 (1980) 553; N. E. Bralić, Phys. Rev. D22 (1980) 3090; P. M. Fishbane, S.Gasioroviwcz,P. Kraus, Phys. Rev. D24 (1981) 2324; Yu. Simonov, Sov. J. Nucl. Phys. 48 (1988) 512.

[35] N. G. Van Kampen, Physica 74 (1974) 215,239; Phys. Rep. C24 (1976) 172.

[36] A. Yu. Dubin, Yu. S. Kalashnikova, Phys. Atom. Nucl. 58 (1995) 1967.

[37] E. Eichten, K. Gottfried, T. Kinoshita, K. D. Lane, T. M. Yan: Phys. Rev. D21 (1980) 203.

[38] G. Barbiellini et al., Phys. Lett. 39B (1972) 663;

G. Giacomelli, M Jacob, Phys. Rep. C55, (1979) 1.

[39] M. M. Block, R. N. Cahn, Rev. Mod. Phys. 57, (1985) 563.

[40] P. V. Landshoff, Phys. Rev. D10 (1974) 1024.

[41] A. Donnachie, P. V. Landshoff, Z. Phys. C2 (1979) 55; Phys. Lett. 123B (1983) 345.

[42] The L3 Collaboration, CERN-PPE/97-48 (1997)

[43] J. J. Sakurai, Ann. Phys. 11 (1960) 1.

[44] H. Alvensleben et al., Nucl. Phys. B18 (1970) 333;

G. McClellan et al., Phys. Rev. D4 (1971) 2683.

[45] Th. Udem et al., Phys. Rev. Lett. 79 (1997) 2646;

[46] S. R. Amendolia et al., Nucl. Phys. B277 (1986) 168; S. R. Amendolia et al., Phys. Lett. BB178 (1986) 435. 